\title{
Minimal tool set for a prokaryotic circadian clock
}

Nicolas M. Schmelling ${ }^{1}$ (D), Robert Lehmann² ${ }^{2}$ Paushali Chaudhury ${ }^{3}$, Christian Beck², Sonja-Verena Albers ${ }^{3}$ (D), Ilka M. Axmann ${ }^{1 *}$ (i) and Anika Wiegard ${ }^{1}$ (D)

\begin{abstract}
Background: Circadian clocks are found in organisms of almost all domains including photosynthetic Cyanobacteria, whereby large diversity exists within the protein components involved. In the model cyanobacterium Synechococcus elongatus PCC 7942 circadian rhythms are driven by a unique KaiABC protein clock, which is embedded in a network of input and output factors. Homologous proteins to the KaiABC clock have been observed in Bacteria and Archaea, where evidence for circadian behavior in these domains is accumulating. However, interaction and function of non-cyanobacterial Kai-proteins as well as homologous input and output components remain mainly unclear.

Results: Using a universal BLAST analyses, we identified putative KaiC-based timing systems in organisms outside as well as variations within Cyanobacteria. A systematic analyses of publicly available microarray data elucidated interesting variations in circadian gene expression between different cyanobacterial strains, which might be correlated to the diversity of genome encoded clock components. Based on statistical analyses of co-occurrences of the clock components homologous to Synechococcus elongatus PCC 7942, we propose putative networks of reduced and fully functional clock systems. Further, we studied KaiC sequence conservation to determine functionally important regions of diverged KaiC homologs. Biochemical characterization of exemplary cyanobacterial KaiC proteins as well as homologs from two thermophilic Archaea demonstrated that kinase activity is always present. However, a KaiA-mediated phosphorylation is only detectable in KaiC1 orthologs.

Conclusion: Our analysis of 11,264 genomes clearly demonstrates that components of the Synechococcus elongatus PCC 7942 circadian clock are present in Bacteria and Archaea. However, all components are less abundant in other organisms than Cyanobacteria and KaiA, Pex, LdpA, and CdpA are only present in the latter. Thus, only reduced KaiBC-based or even simpler, solely KaiC-based timing systems might exist outside of the cyanobacterial phylum, which might be capable of driving diurnal oscillations.
\end{abstract}

Keywords: Cyanobacteria, Archaea, Circadian clock, BLAST, Kinase activity, Output factors, Input factors, Length distribution, Co-occurrence

\section{Background}

Life on Earth is under the influence of changing environmental conditions, which not only pose a challenge to organisms, but also present a chance of adaptation and therefore a possible fitness advantage over competitors $[1,2]$. Using inner timing systems organisms can coordinate their physiology and behavior according to the daily

\footnotetext{
*Correspondence: Ilka.Axmann@hhu.de

'Institute for Synthetic Microbiology, Cluster of Excellence on Plant Sciences

(CEPLAS), Heinrich Heine University Duesseldorf, Universitaetsstrasse 1, 40225 Duesseldorf, Germany

Full list of author information is available at the end of the article
}

recurring changes. Simple timing systems work in an hour glass like fashion and need to be reset every day by environmental stimuli, whereas true circadian clocks generate self-sustained and temperature-compensated 24-hour rhythms of biological activities [3, 4].

Circadian clocks are found in many eukaryotes such as algae, plants and mammals [5]. Even though circadian clocks seem like a conserved trait in evolution, differences in the protein components, involved in circadian timing, suggest a convergent evolution of timing mechanisms [5]. For many years it has been believed that something as complex as a circadian clock could not have been evolved 
in unicellular organisms like prokaryotes [5-7]. However, the existence of temperature compensated 24-hour rhythms of cell division in Synechococcus sp. WH 7803 and circadian nitrogen fixation in Cyanothece sp. PCC 8801 proved otherwise [8-12]. The molecular basis of the cyanobacterial circadian clock was intensively investigated in Synechococcus elongatus PCC 7942 (hereafter Synechococcus 7942), where the core clockwork resembles a posttranslational oscillator [13-15]. In contrast, eukaryotic circadian rhythms are believed to be mainly based on transcriptional-translational feedback loops. However, findings on post-translational systems are accumulating [16-18] and might exist also in Archaea [16].

Light is assumed to be the driving stimulus in circadian clock entrainment [19]. In Synechococcus 7942, contrary to eukaryotic circadian clock systems, a photoreceptor in the input pathway of the clock could not be detected thus far. Instead, Synechococcus 7942 cells sense light indirectly through the redox and energy state of the cell [20]. Here, two metabolic components are considered to play a major role [21]: The ATP to ADP ratio and the redox state of the plastoquinone pool $[22,23]$. The core of the circadian clock in Synechococcus 7942 consists of three proteins KaiA, KaiB and KaiC. KaiC monomers are composed of two domains, which assemble into two hexameric rings $[24,25]$. The C-terminal ring is capable of autophosphorylation and -dephosphorylation [26, 27]. KaiC phosphorylation is stimulated by the interaction with KaiA $[28,29]$, and additionally, affected by the ATP/ADP ratio of the cell [30]. KaiB inhibits the activating effect of KaiA and initializes dephosphorylation [31]. Altogether, KaiC hexamers phosphorylate and dephosphorylate rhythmically during the course of a day. Two very recent studies provide a structural basis for the dynamic assembly of clock proteins by using crystallography and NMR [32], and mass spectrometry and cryo-electron microscopy of the native proteins [33]. The binding of oxidized quinones to KaiA has been suggested to stop the clock directly by causing KaiA aggregation [34, 35]. The KaiABC core clock is embedded into a network of input and output pathways. The input factors that interact with the core clock are Pex, LdpA, PrkE, NhtA, IrcA, CdpA [20, 36-40]. Output factors are SasA, LabA, LalA, CpmA, Crm, RpaA, and RpaB, as well as CikA, which is functioning both in input and output pathway of the circadian clock [41-53].

Sequence analysis indicated that at least three different types of timing systems are present in Cyanobacteria, (i) a KaiABC-based system as in Synechococcus 7942, (ii) a reduced system with a KaiBC core and a reduced set of input/output factors as in Prochlorococcus and (iii) a reduced KaiABC system as in Synechococcus sp. WH 8102, which despite including all three kai genes, has the same input/output factors as the reduced KaiBC system [54]. Furthermore, multiple kai genes can exist in an organism
[55, 56]. In Synechocystis sp. PCC 6803 (hereafter Synechocystis 6803) for example, three copies of both kaiB and $\mathrm{kaiC}$ are found. KaiA, KaiB1 and KaiC1, most similar to the kai genes of Synechococcus 7942 [57, 58], are thought to resemble the core clock. KaiB3 and KaiC3 are thought to function as fine tuning factors for the circadian clock in Synechocystis 6803, whereas no circadian function has been found for KaiB2 and KaiC2 [58]. However, recently kaiC2-dependent adaptive growth and diurnal rhythms of nitrogen fixation were observed in Rhodopseudomonas palustris [59]. Homologs of $k a i B$ and $k a i C$ genes also exist in other Bacteria and even Archaea, where a shortend KaiC most often resembles only one domain [55]. An archaeal one-domain KaiC homolog was shown to form a hexameric ring, similar to the two duplicated domains of Synechococcus 7942 KaiC [60]. In Haloferax volcanii the transcripts of four kaiC homologs display diurnal accumulation profiles [61]. However, the function of non-cyanobacterial Kai-proteins is mainly unclear, so far. Although some of the input- and output factors were also found in prokaryotes other than cyanobacteria $[51,54,62,63]$, it is unknown whether the Kai homologs outside the cyanobacterial phylum or the additional cyanobacterial Kai homologs interact with (other) in- and output factors.

In this study, we performed BLAST analyses to first identify possible KaiC-based timing systems in organisms outside of Cyanobacteria and second to explore variations in circadian clocks within Cyanobacteria. Further, we examined variations in circadian gene expression between different cyanobacterial strains using microarray data. Together, this aims at decoding the correlation between Kai proteins and additional clock components. Based on the co-occurrence of clock components known from Synechococcus 7942 we propose putative networks of reduced and fully functional clock systems. Further, we used the sequence information of $\mathrm{KaiC}$ and its homologs in Cyanobacteria to determine the similarities at important sites of the protein. We chose cyanobacterial KaiC proteins as well as homologs from two thermophilic Archaea and demonstrated that kinase activity is always present. However, a KaiA-mediated phosphorylation is only detectable in cyanobacterial KaiC1 homologs.

\section{Methods}

\section{Programming languages}

The programming languages Python (version 3.5.1) and $\mathrm{R}$ (version 3.2.3) were used in this work. The processing and analysis of the microarray time series datasets was performed using R. Regarding the distribution analysis, the Biopython project ([64]; version 1.66) was used to download from GenBank as well as to work with FASTA files. Besides Biopython, the Python packages: IPython ([65] version 4.1.1) as an interactive Python 
environment with the IPython notebook; numpy and scipy ([66]; version 1.10.4, version 0.17.0) for numerical operations; matplotlib ([67]; version 1.5.1) for data visualization; and pandas ([68]; version 0.17.1) for data analyses were used. The Python code necessary to reproduce the BLAST analyses is available on https://github.com/ schmelling/reciprocal_BLAST GitHub (http://doi.org/10. 5281/zenodo.229910) [69].

\section{Reciprocal BLAST and NCBI}

The coding sequences of all entries in the genbank protein database [70], which were labeled as "Complete Genome" or "Chromosome", were downloaded from the NCBI FTP server (version May 2016). These sequences, including the coding sequences of Synechococcus elongatus PCC 7942 and Synechocystis sp. PCC 6803, were used to construct a custom protein database for the homology search. Further, protein sequences of the 23 clock related proteins (Additional file 1: Table S1), from Synechococcus elongatus PCC 7942 and Synechocystis sp. PCC 6803, respectively, were checked against the entries in the Cyanobase Database to ensure correctness [71] (version May 2016). These 23 protein sequences were used as queries for a search of homologs within the custom protein database, applying the standalone version of BLASTP 2.2.30+ [72] (May 2016, dx.doi.org/10.17504/protocols.io. grnbv5e) with standard parameter (wordsize: 3 , substitution matrix: BLOSUM62). The 10,000 best hits with an e-value of $10^{-5}$ or lower were filtered for further analyses. The first BLAST run returned circa 65,000 hits for all 23 cyanobacterial proteins combined.

These hits were used as queries for a second reverse BLASTP run, searching for homologs in Synechococcus 7942 or Synechocystis 6803 genomes using the same parameters as above with an altered e-value of 10. Only hits with the original query protein as best reversal hit were accepted for further analyses, thus minimizing false positive results.

Raw and processed data is available on https://figshare. com/authors/Nicolas_Schmelling/699391 figshare (https:// dx.doi.org/10.6084/m9.figshare.3823902.v3, https://dx.doi. org/10.6084/m9.figshare.3823899.v3) [73, 74].

\section{Testing of co-occurence}

Co-occurrence of circadian clock proteins was examined by using the right-sided Fisher's exact test [75]. For each of the 94 cyanobacterial strains, all identified homologous clock genes were gathered into one set. The phylogenetic distribution of Cyanobacteria in the NCBI genbank database is very imbalanced. Some genera (e.g. Prochlorococcus and Synechococcus) are covered better than others. To avoid selection bias, we removed sets with identical combinations of genes, resulting in 69 unique clock systems. Null hypothesis of Fisher's exact test is a pairwise independent distribution of the proteins across all clock systems. P-values were corrected for multiple testing after Benjamini-Hochberg [76] with an excepted false discovery rate of $10^{-2}$. We denote that due to the nature of statistical testing, proteins appearing in almost all clock systems are always virtually independent to others. All proteins were clustered according to their corrected p-values.

\section{Microarray analysis}

The diurnal expression program of six cyanobacterial strains was probed using microarray time series datasets (Additional file 1: Table S3). Unfortunately, the data for the two reported Synechocystis 6803 experiments by Labiosa and colleagues [77] and Kucho and coworkers [78] could not be obtained. The study by Toepel and coworkers [79] had to be discarded due to the employed ultradian light cycles (6:6 LD cycles). The Synechocystis 6803 datasets, the Synechococcus 7942 dataset of Ito and colleagues, and the Anabaena sp. PCC 7120 dataset were $12 \mathrm{~m}$ transformed, while the Cyanothece ATCC 51142 datasets are only available after transformation. The two biological replicates of the Anabaena sp. PCC 7120 dataset were concatenated for the following analyses [80], similar to the Synechocystis 6803 dataset [81]. Expression profiles were smoothed using a Savitzky-Golay lowpass filter, as proposed by Yang and colleagues [82], in order to remove pseudo peaks prior to the detection of periodic genes. Diurnally oscillating expression profiles were detected using harmonic regression analysis [83]. The derived p-values for each gene is based on the assumption of a linear background profile as compared to the sinoidal foreground model. After multiple hypothesis testing correction according to Benjamini-Hochberg [76], all datasets yielded significantly oscillating genes $(\mathrm{q} \leq 0.05)$ except for Microcystis aeruginosa PCC 7806, with 7 samples the shortest dataset.

\section{Multiple sequence alignment}

Multiple sequence alignments were constructed by the standalone version of CLUSTAL Omega 1.2.1-1 [84] using 20 iterations, while only one iteration was used to construct the guide tree (May 2016, dx.doi.org/10.17504/ protocols.io.gscbwaw). The sequences for the alignments were obtained from the processed data generated, as described in the method section "Reciprocal BLAST and NCBI". Afterwards the alignments were adjusted to Synechococcus 7942 sequence with Jalview [85] and edited multialignments were used to create WebLogos [86].

\section{Cloning, heterologous expression and purification of Kai proteins}

To express KaiC1 proteins from Synechocystis sp. PCC 6714, Nostoc punctiforme ATCC 29133, Cyanothece sp. PCC 7424 as well as KaiC3 from Cyanothece sp. PCC 7424, 
Microcystis aeruginosa PCC 7806, Pycrococcus horikoshii OT3 PH0833, and Thermococcus litoralis DSM5473, the respective $\mathrm{kaiC}$ genes were amplified by PCR from genomic wildtype DNA. The ORFs and primers are listed in Additional file 1: Table S4. Amplified sequences were ligated into BamHI and NotI restriction sites of the plasmid pGEX-6P-1 (GE Healthcare). For P.horikoshii and T.litoralis KaiC3 the amplified PCR products were ligated into pETDuet1 Vector using BamHI/HindIII and PstI/HindIII restriction enzymes in MCS1, respectively. Escherichia coli DH5 $\alpha$ or BL21 (DE3) cells were transformed with the resulting plasmids (pGEX-kaiC1Sy6714, pGEX-kaiC1-Npun29133, pGEX-kaiC1-Cy7424, pGEX-kaiC3-Cy7424, pGEX-kaiC3-Mic7806, pSVA3151, pSVA3152). For expression of GST-fused KaiA-7942 and KaiC-7942 pGEX derivatives, kindly provided by T. Kondo (Nagoya University, Japan), were used. Expression of GSTKai proteins occurred at $37{ }^{\circ} \mathrm{C}$ and $200 \mathrm{rpm}$ in Terrific broth medium containing $100 \mu \mathrm{g}$ ampicillin $\mathrm{ml}^{-1}$. GST-KaiA-7942 expression was induced with $1 \mathrm{mM}$ isopropyl $\beta$-D-thiogalactopyranoside (IPTG) and carried out overnight, whereas GST-KaiC homologs were expressed for 72 hours without induction. Cells were harvested and lysed in ice-cold extraction buffer $(50 \mathrm{mM}$ Tris/ $\mathrm{HCl}$ (pH 8), $150 \mathrm{mM} \mathrm{NaCl}, 0.5 \mathrm{mM}$ EDTA, $1 \mathrm{mM}$ DTT, $5 \mathrm{mM} \mathrm{MgCl} 2$ and $1 \mathrm{mM}$ ATP). Recombinant GST-Kai proteins were affinity purified using Protino Gluthatione Agarose 4B (Macherey-Nagel) as described in Wiegard and colleagues [57]. During the procedure the GST-tag was removed with PreScission protease in cleavage buffer (50 mM Tris/HCl (pH 8), $150 \mathrm{mM} \mathrm{NaCl}, 1 \mathrm{mM}$ EDTA, 1 $\mathrm{mM}$ DTT, $5 \mathrm{mM} \mathrm{MgCl}$ and $0.5 \mathrm{mM}$ ATP). Homogeneity of the recombinant proteins was controlled by separating them via SDS-PAGE. If it was not sufficient, proteins were further purified by anion-exchange chromatography using a MonoQ 5/50 GL or ResourceQ column (GE Healthcare). After dialysis in reaction buffer $(20 \mathrm{mM}$ Tris/ $\mathrm{HCl}$ (pH 8), $150 \mathrm{mM} \mathrm{NaCl}, 0.5 \mathrm{mM}$ EDTA, $5 \mathrm{mM} \mathrm{MgCl}_{2}$, and $1 \mathrm{mM}$ ATP) protein concentration was determined using infrared spectroscopy (Direct detect, Merck Millipore). Proteins were stored at $-20{ }^{\circ} \mathrm{C}$. For His-tagged KaiC3 homologs, E. coli BL21 (DE3) RIL cells were transformed with pSVA3151 (P.horikoshii) and pSVA3152 (T.litoralis), and grown as preculture overnight at $37^{\circ} \mathrm{C}$ in LB medium containing ampicillin $\left(50 \mu \mathrm{g} \mathrm{ml}^{-1}\right)$ and chloramphenicol $\left(34 \mu \mathrm{g} \mathrm{ml}^{-1}\right)$. Fresh medium containing antibiotic was inoculated with $0.1 \%$ preculture and grown at $37{ }^{\circ} \mathrm{C}$ to an $\mathrm{OD}_{600}$ of 0.7. After induction with $0.3 \mathrm{mM}$ of IPTG, growth was continued for 16 hours at $16{ }^{\circ} \mathrm{C}$. Cells were collected by centrifugation, frozen in liquid nitrogen, and, after storage at $-80^{\circ} \mathrm{C}$, resuspended in $50 \mathrm{ml}$ lysis buffer (50 mM Hepes- $\mathrm{NaOH}, \mathrm{pH} 7.2,150 \mathrm{mM} \mathrm{NaCl}$ ) containing complete EDTA-free protease inhibitor cocktail (Roche) together with DNase I and lysed by sonication. Cell debris were removed by centrifugation at $4{ }^{\circ} \mathrm{C}$ for $30 \mathrm{~min}$ at $20,000 \mathrm{x}$ g. Further, Ni-NTA (Sigma Aldrich, Seelze, Germany) based purification was performed using columns equilibrated in purification buffer $(50 \mathrm{mM}$ Hepes- $\mathrm{NaOH}, \mathrm{pH} 7.2,150 \mathrm{mM} \mathrm{NaCl})$. For the removal of unspecifically bound proteins, columns were washed with 15 column volumes of equilibration buffer including 10 $\mathrm{mM}$ imidazole. T.litoralis KaiC3 was eluted in the same buffer with $150 \mathrm{mM}$ imidazole containing equilibration buffer, whereas for P.horikoshii KaiC3 the elution was carried out in $20 \mathrm{mM}$ MES pH 6.2, $150 \mathrm{mM} \mathrm{NaCl}, 150 \mathrm{mM}$ imidazole as this protein is stable in low pH buffer. Further purification of T.litoralis KaiC3 was achieved by size exclusion chromatography using Superdex 200 10/300 GL. P.horikoshii $\mathrm{KaiC} 3$ was incubated at $50^{\circ} \mathrm{C}$ for $20 \mathrm{~min}$ and centrifuged for $15 \mathrm{~min}$ at $10.000 \mathrm{x} \mathrm{g}$. Subsequently, the supernatant was dialyzed overnight against $20 \mathrm{mM}$ MES pH 6.2, $150 \mathrm{mM} \mathrm{NaCl}$ buffer. As a quality control, proteins were separated via SDS-PAGE and pure proteins were frozen in liquid nitrogen and kept at $-80^{\circ} \mathrm{C}$.

\section{In vitro phosphorylation assays}

To investigate KaiA dependent phosphate uptake 12 $\mu \mathrm{g}$ of KaiC-7942, KaiC1-Sy6714, KaiC1-N29133, KaiC1Cy7424, KaiC3-Cy7424, KaiC3-Mic7806, KaiC3-T.lit or KaiC3-P.hor were mixed with $10 \mu \mathrm{Ci} \gamma$-P ${ }^{32}$-ATP in $60 \mu \mathrm{l}$ Tris reaction buffer $(20 \mathrm{mM}$ Tris/ $\mathrm{HCl}(\mathrm{pH} 8), 150 \mathrm{mM}$ $\mathrm{NaCl}, 0.5 \mathrm{mM}$ EDTA, $5 \mathrm{mM} \mathrm{MgCl} 2,1 \mathrm{mM}$ ATP) in the presence or absence of $6 \mu \mathrm{g}$ KaiA-7942. $10 \mu \mathrm{l}$ aliquots were taken after $0,0.75,1.5,3$ and 22 hours of incubation at $30^{\circ} \mathrm{C}$ and reaction was stopped by adding SDS-sample buffer. Proteins were separated in high-resolution polyacrylamide gels $(10 \% \mathrm{~T}, 0.67 \% \mathrm{C})$ by SDS-PAGE (modified from [87]), stained with Coomassie brilliant blue and subjected to autoradiography. Signals were analyzed using a Fujifilm FLA-3000 (FUJIFILM). To analyze in vitro phosphorylation of KaiC3-T.lit and KaiC3-P.hor at higher temperatures, $10 \mu \mathrm{g}$ of the recombinant proteins were incubated with $10 \mu \mathrm{Ci} \gamma$-P $\mathrm{P}^{32}$-ATP in $50 \mu \mathrm{l}$ HEPES reaction buffer ( $50 \mathrm{mM}$ HEPES (pH 7.2), $150 \mathrm{mM} \mathrm{NaCl}, 5$ $\mathrm{mM} \mathrm{MgCl} 2,1 \mathrm{mM}$ ATP) or $50 \mu \mathrm{l}$ MES reaction buffer (50 mM MES (pH 6), $150 \mathrm{mM} \mathrm{NaCl}, 5 \mathrm{mM} \mathrm{MgCl}$, $1 \mathrm{mM}$ ATP), respectively, at $75{ }^{\circ} \mathrm{C}$. After $0,5,10$ and 15 minutes $10 \mu \mathrm{l}$ aliquots were taken and analyzed by SDS-PAGE and autoradiograpy as described above. Comprehensive protocols are available on protocols.io (dx.doi. org/10.17504/protocols.io.g3gbyjw, dx.doi.org/10.17504/ protocols.io.gysbxwe).

\section{Results and discussion}

\section{Most complete set of circadian clock orthologs in} Cyanobacteria

Growing evidence hints at circadian rhythms in many Cyanobacteria and first sequence analyses revealed that 
the core genes, known from Synechococcus 7942, are conserved in almost all cyanobacterial species $[4,55,57,88]$. Even though daily rhythms seem to be conserved in Cyanobacteria, composition and quantity of corresponding genes on the genome level show high variability [56]. Dvornyk and colleagues first attempted to describe the variety of cyanobacterial core circadian clock systems in 2003 [55]. Since then, the amount and depth of sequencing data increased manifold, which allowed us to perform a detailed analysis of the KaiC-based circadian clock including the input and output pathways. The circadian clock proteins of Synechococcus 7942 (Additional file 1: Table S1) and the three diverged KaiB and KaiC homologs from Synechocystis 6803 (Additional file 1: Table S1) were the basis for our analysis. Their protein sequences served as basis for a reciprocal best hit BLAST analysis. Organisms with at least one homolog to $\mathrm{KaiC}$ were retained for further analysis. This stringent filter was essential since KaiC represents the core of the circadian clock in Synechococcus 7942. These organisms were grouped by their corresponding genus for a first overview of the homolog distribution (Fig. 1a). We found homologs in Cyanobacteria, Proteobacteria, Archaea, as well as other Bacteria such as Chloroflexi. This finding is in good agreement with previous studies [55, 57, 89]. However, our comprehensive study identified a plethora of new bacterial and archaeal genera harboring homologs to the circadian clock genes (Fig. 1a). Nevertheless, Cyanobacteria represent the phylum with the highest degree of sequence similarity and integrity of the system followed by Bacteria, mostly Proteobacteria. In Archaea, homologs to only a fraction of core genes could be identified (Fig. 1a).

\section{Core circadian clock factors KaiB and KaiC beyond the phylum of Cyanobacteria}

Four out of seventeen studied factors are exclusively found in Cyanobacteria (Fig. 1a). One of these factors is KaiA, as previously reported in studies with a smaller sample size [55, 89]. However, even some Cyanobacteria, like Candidatus Atelocyanobacterium thalassa (previously named Cyanobacterium UCYN-A), and all representatives from the genus Prochlorococcus lack kaiA (Fig. 1a) [56]. Interestingly, multiple copies of kaiA in a single cyanobacterial genome could not be identified. This is of special interest, because we could observe strong sequence length variations for KaiA (Fig. 5a, c) and multiple copies for the other core proteins $\mathrm{KaiB}$ and KaiC have been reported (Fig. 1b) [55-57]. With the kaiAlacking Candidatus Atelocyanobacterium thalassa and a kaiA-containing cyanobacterial endosymbiont [90] only two out of 94 studied Cyanobacteria do not contain a $k a i B$, whereas a third of the cyanobacterial genera contain multiple copies of $k a i B$ and $k a i C$ (Fig 1b, c). There are a few exceptional Cyanobacteria like Gloeobacter violaceus
[91], which are even lacking the $\mathrm{kaiC}$ gene and are thus not detected in this analysis due to the previously described filtering criteria. However, all Cyanobacteria having homologs to $\mathrm{kaiB}$ and $\mathrm{kaiC}$ are coding for at least one pair of proteins most similar to KaiB1 and KaiC1 from Synechocystis 6803 (Fig. 1b, c), which is consistent with previous studies [55, 57]. Only Cyanothece sp. PCC 7822 has homologs similar to all three kaiB and kaiC copies from Synechocystis 6803 (Fig. 1c). The majority (67.68\%) of the bacterial genera, outside of the cyanobacterial phylum, encode KaiC3-like proteins. Approximately half of them also contain additional KaiC homologs, most similar to $\mathrm{KaiC} 1, \mathrm{KaiC} 2$, or sometimes even both (Fig. 1b). This observation does not hold true for KaiB homologs, here KaiB2 is the major KaiB homolog (75.86\%). One group of organisms, all belonging to the phylum of Bacteroidetes, stands out in this analysis, because they encode only proteins similar to KaiB2/KaiC2 (Fig. 1b). Archaeal genera show mainly homologs with highest identity to $\mathrm{KaiC} 1$ or KaiC3. Furthermore, almost all of the Archaea have multiple copies of the KaiC protein. Whereas only four have homologs to $\mathrm{KaiB}$, which is either similar to KaiB2 or in thermophilic Methanothermobacter similar to KaiB1 (Fig. 1b).

\section{Circadian clock factors solely involved in cyanobacterial input pathways}

Multiple clock input factors are present in all four taxonomic groups namely PrkE, NhtA, and CikA. The latter also acts in the output of the circadian clock (Additional file 1: Table S2, Fig. 1a) $[38,39,46]$. The prevalence of CikA is in good agreement with its tremendous effect on resetting the circadian clock in Synechococcus 7942 [39], where its interaction with the Kai complex is mediated by additional proteins $[38,40]$. CikA destabilizes in the presence of oxidized quinones and thereby integrates information about the cellular redox state into the oscillator [38]. Naturally, CikA-lacking Prochlorococcus are not able to reset their timing mechanism in continuous light [92], pointing at the importance of this factor. However, single mutations in sasA can restore circadian properties in Synechococcus 7942 cikA mutants and it has been suggested that a simpler network with modified interactions of the other clock proteins can exist [93].

The input factors Pex, LdpA, and CdpA are found to be unique for Cyanobacteria (Fig. 1a), confirming a previous analysis of $l d p A$ [94]. Hence, with Pex, a transcriptional repressor of kaiA [36], and LdpA, a redox-sensing protein [20] two factors, which sense the cellular metabolic state of the circadian clock in Synechococcus 7942, are missing outside of the cyanobacterial phylum. LdpA is also the only input factor present in the reduced but functional timing system of Prochlorococcus [56, 88]. This might indicate the necessity of this factor for the entrainment 


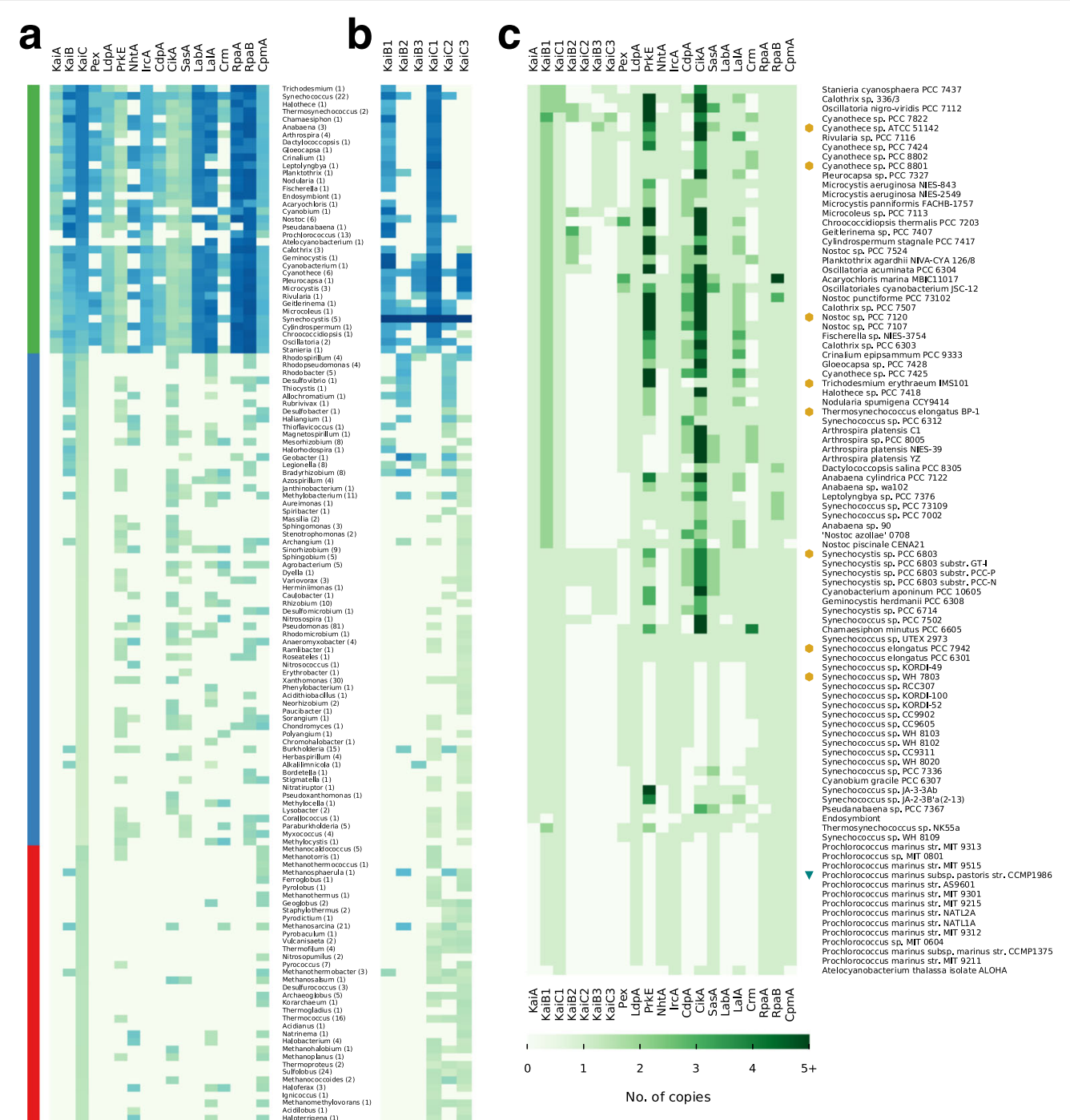


Fig. 1 (See figure on previous page)

Distribution of circadian clock proteins. $\mathbf{a}$, b Shown are the mean sequence similarites of each protein for each genus that contains a KaiC homolog from Synechococcus 7942. The genera are sorted by their group and KaiC similarity. The number in parenthesis represents the number of individual genomes per genus. a shows the mean similarities for the circadian clock proteins of Synechococcus 7942. b shows the mean similarities for the circadian clock proteins of Synechocystis 6803. The four taxonomic main groups are highlighted: Cyanobacteria (Green), Proteobacteria (Blue), Archaea (Red), Other (Grey). c shows the number of homologs for each protein in each cyanobacterium. Copy numbers higher than five were condensed. Cyanobacterial strains with true circadian clocks [8, 78-80, 106, 143-148] are highlighted with a yellow hexagon and the strain with hourglass like timing mechanism [92] with a green triangle. We could not find references for all strains, however, we identified strains with circadian rhythms, which are not listed here as their genomes are still not fully assembled

of the clock [62]. Nevertheless the possibility of a functional clock without Pex and LdpA remains, since LdpA and Pex mutants in Synechococcus 7942 are only altered in their period length $[37,95]$ and Pex is also missing in the KaiA-lacking Prochlorococcus and the KaiA-containing Synechocystis 6803 (Fig. 1a). In Synechococcus 7942 the third unique cyanobacterial input factor, CdpA, influences phase resetting and acts in parallel to CikA [40]. Since CdpA seems to be essential in Synechococcus 7942 [40], it likely has a prior role in processes other than phase resetting and is hence dispensable for input pathways in other organisms than Cyanobacteria.

Altogether, the absence of three important input factors outside of the cyanobacterial phylum suggests that other entrainment systems might be used for putative timing systems. In Rhodobacter sphaeroides, which displays circadian gene expression rhythms, a histidine kinase is encoded in an operon with kaiBC and was suggested as a candidate for transducing the redox signal to KaiBC [96]. Further, the direct entrainment by the ATP/ADP ratio $[30,97]$ might be the primary mechanism to synchronize the circadian clock with metabolism and the environment.

\section{Central output factors are missing in Archaea and non-cyanobacterial genera}

The output pathway of the circadian clock in Synechococcus 7942 involves eight proteins (see Fig. 7). RpaA serves as a key regulator [45]. Its activity is indirectly modulated depending on the phosphorylation state and the ATPase activity of KaiC [46, 98]. SasA (antagonistically to CikA) connects the core clock to RpaA, which in turn regulates global gene expression, including the $\mathrm{kaiBC}$ promoter [43, 45, 46, 99]. LabA, Crm and RpaB are also known to affect RpaA [44, 48, 49, 52, 99]. CpmA modulates kaiA expression by an unknown mechanism [51]. In contrast to the unique cyanobacterial input factors, we found none of the eight output proteins exclusively in Cyanobacteria. Homologs of five factors (SasA, CikA, LalA, Crm, CpmA) are present in all four investigated taxonomic groups (Additional file 1: Table S2). CpmA is a member of a superfamily essential for purine biosynthesis and thus likely to have orthologs in other organisms [51]. However, RpaA and RpaB are not present in Archaea and SasA is only found in the methanogenic genera
Methanospirillum, and Methanosalsum. Hence, the entire central output pathway is missing in Archaea (Fig. 1a). In addition, orthologs for RpaA are found in only nine non-cyanobacterial genera, questioning whether another transcription factor might read out the putative core timer in other Bacteria (Fig. 1a). A previous BLAST search by Dvornyk and colleagues revealed that SasA homologs in non-cyanobacterial prokaryotes lack the KaiB-like domain [63]. This finding is confirmed in our analysis, indicating that stimulations of SasA homologs by KaiC outside of Cyanobacteria are very unlikely, because interaction occurs via this KaiB-like domain, which adopts a thioredoxin-like fold [41, 100]. Altogether, our analysis reveals that possible circadian clocks of Bacteria and Archaea must use an output pathway that is different from the one described in Synechococcus 7942.

\section{Co-occurrence analysis hints at the core module for circadian timing}

The previous analysis revealed substantial differences in the composition of the clock components between Cyanobacteria, other Bacteria, and Archaea. Even within Cyanobacteria there is a huge variety in the composition of the potential circadian clocks (Fig. 1c). Cyanobacteria have either a severely reduced timing systems, such as the one in Prochlorococcus, a standard system as seen in Synechococcus 7942, or an inflated system as found in Synechocystis 6803 (Fig. 1c). This trichotomy of systems raises questions about essentiality and pairwise cooccurrence of circadian clock proteins. These questions were answered in a series of right-sided Fisher's exact tests. To avoid systematic biases due to an overrepresentation of closely related strains [101], we extracted 69 unique combinations of the 21 circadian clock factors as described in the Methods section.

Within these 69 unique systems two factors are always present: (i) KaiC, because we selected for organisms containing at least one KaiC-like protein and (ii) RpaB, which is associated with cell size and circadian gene expression $[44,53]$. RpaB competes for promoter binding sites with RpaA and its phosphorylated state is thought to inhibit the phosphorylation of RpaA [49]. Other factors present in the majority ( $\geq 90 \%$ ) of the observed unique clock systems are KaiA, KaiB, LdpA, IrcA, CikA, SasA, RpaA and 


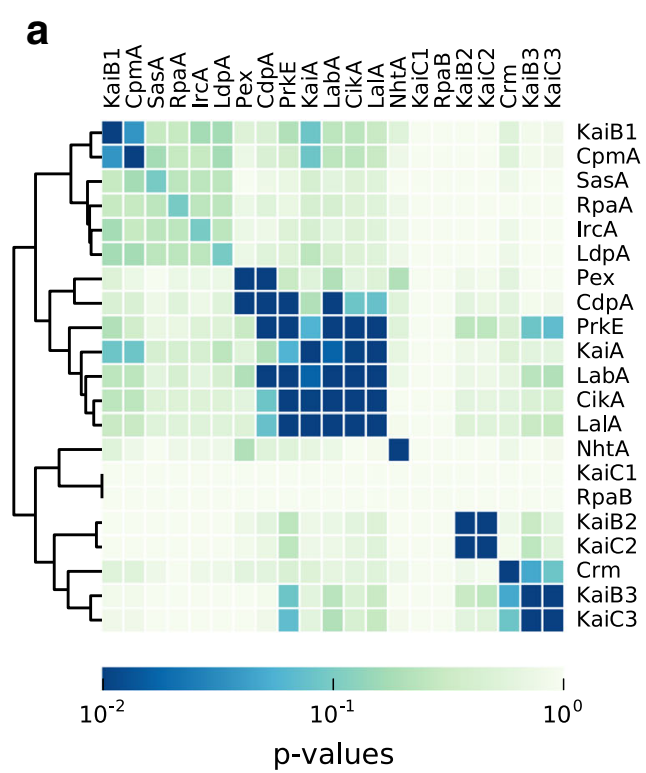

\section{b}
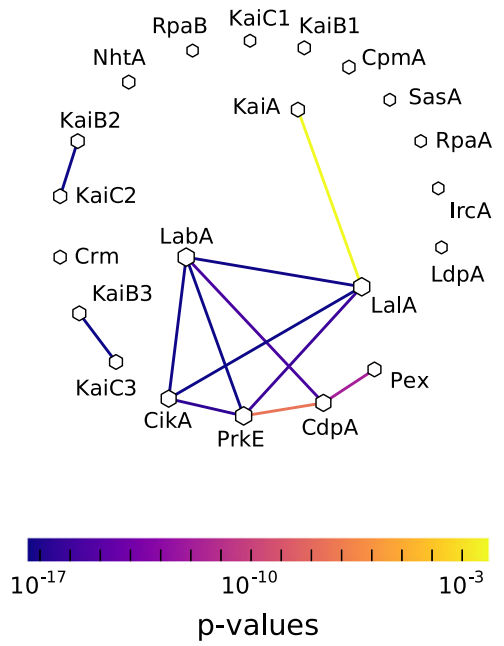

Fig. 2 Co-occurrence of circadian clock proteins in Cyanobacteria. a The $p$-values, calculated by pair-wise Fisher's exact tests, are visualized in a heatmap. Only $p$-values $\leq 0.01$ are considered as significant. Proteins are sorted by a hierarchical agglomerative clustering algorithm. b Network of significant co-occurring circadian clock factors in Cyanobacteria, calculated in regard to the results of the pair-wise Fisher's exact test. The line color corresponds to the level of significance. Missing links are those that had a higher $p$-value than 0.01 . Node size is proportional to the degree of that node

CpmA. Because of their abundance, most of these factors show no pairwise co-occurence. For example RpaA and RpaB are found in 68 and all 69 clock systems, respectively. Thus their joint presence comes to no surprise. Instead, the finding confirms essential roles in global transcription regulation of Cyanobacteria [102]. With Fisher's exact test we seek to identify gene pairs rather unexpectedly cooccuring in a smaller subset of organisms. Such findings can indicate a common function in the circadian clock system. Only KaiA and CikA, out of the most abundant factors, show significant co-occurence with other factors.

Within the input pathway we detected three siginficantly co-occuring pairs (Fig. 2): (i) CikA and its interaction partner PrkE [40] (ii) PrkE and CdpA and (iii) the kaiA repressor Pex and CdpA. The first two results are in good agreement with a previous study [40]. Within the output pathway, there is a significant co-occurrence between LabA and its ortholog LalA. Interestingly, we identified several significant co-occurrences between factors of the input and the output pathway. CikA, which functions in the input and output of the clock, co-occurs significantly with LabA, and LalA (Fig. 2). This fits well in the overall picture as CikA and LabA are thought to regulate the activity of RpaA [46,52]. Additionally, PrkE shows also significant occurrences with both LabA and LalA (Fig. 2). Furthermore, CdpA was found to co-occur significantly with LabA. (Figure 2) This is of special interest since PrkE and CdpA are only known as interaction partners of CikA, and both are involved in phase resetting, and cell division, respectively [40]. This result, however, hints at potential increased involvement of PrkE and CdpA in the RpaA regulation and supports the view of an integrated network with overlapping interactions of input and output factors [103]. Notably, no co-occurrence of NhtA and LdpA was detected, although it was suggested that NhtA might be involved in assembly of the iron-sulfur cofactor of LdpA [40]. Significant co-occurrences with core factors could only be observed between KaiA and LalA (Fig. 2). However, KaiA shows a strong, but not significant, co-occurrence with CikA ( $p=0.0105)$. Lastly, we also found significant co-occurrences between KaiB2 and $\mathrm{KaiC} 2$ as well as KaiB3 and KaiC3 (Fig. 2). This indicates two distinct function of the two pairs. In this context it is worth mentioning that kaiB2-containing archaeal genomes always encode a KaiC2 homolog.

In summary, we identified a conserved set of factors (Fig. 2), both in input and output that show significant co-occurrences. This set, composed of KaiA, PrkE, CdpA, CikA, LabA, and LalA, is found in Cyanobacteria with a true circadian clock such as Synechococcus 7942 and Synechocystis 6803 [78] but is missing in cyanobacterial strains with reduced timing mechanisms such as Prochlorococcus. Additionally, PrkE, CikA, LabA, and LalA are also missing in most marine Synechococcus species. This 
finding hints at the importance of these factors for the functionality of a circadian clock. On the other hand, NhtA and Crm seem to play only a minor or extending role in clock regulation, because they are neither always present nor show significant co-occurrence with other factors.

\section{A systematic analysis of circadian expression in Cyanobacteria}

Genome-wide time-resolved expression measurements in a range of cyanobacterial strains have repeatedly indicated substantial fractions of genes with circadian regulation patterns [104-106]. Considering that all Cyanobacteria share the challenge of a photoautotrophic lifestyle, which requires major changes in the metabolism between day and night, one might expect a common transcriptional regulatory pattern. Thus, we compared a total of nine published microarray time-series datasets of different cyanobacterial strains under constant light or diurnal light conditions (for details see Additional file 1: Table S3), which were available and applicable for this analysis. Not all of the chosen microarray experiments were conducted under constant light conditions, which leads to a combination of circadian-clock regulated and light-induced genes. We therefore refer to genes with oscillating expression as diurnally regulated instead of circadian. For allowing a direct comparison, we reprocessed the raw-data and subjected the resulting expression time series to a harmonic regression oscillation detection. This method assumes a sinusoidal shape of circadian expression profiles and uses linear expression profiles as background, yielding estimates of the peak phase and amplitude of each gene.

In a first step we compared biological replicate datasets to establish the reproducibility of strain-specific circadian expression programs. Similarity between two circadian expression programs was established using the circular correlation coefficient $\rho$ ccc as described by Jammalamadaka and SenGupta [107] applied to estimated peak expression phases. The following analyses were limited to genes with oscillating expression profiles in both compared datasets since only in these cases phase and amplitude estimates are meaningful descriptors. Direct comparison of the oscillation phases and amplitudes indicates good reproducibility between two respective measurements in form of statistically significant elevated correlation of the diurnal expression patterns in Synechococcus $7942(\rho \mathrm{ccc}=0.61, p \ll 0.01)$, Synechocystis 6803 ( $\rho$ ccc $=0.31, p \ll 0.01)$, and Cyanothece sp. ATCC 51142 ( $\rho$ ccc $=-0.51, p \ll 0.01$ ) (Fig 3 top row). While the Synechocystis 6803 datasets show significant similarity, the correlation is diminished by the distinct concentration of expression phases during the day in the beck14 [108] dataset compared to the leh13 [81] measurements.
Interestingly, both Cyanothece sp. ATCC 51142 datasets exhibit a good agreement of peak expression phases with large early day and early night clusters, but the large negative correlation emphasizes the presence of a significant number of anti-phasic gene pairs. The corresponding oscillation amplitude values exhibit high statistically significant correlations $(\rho \mathrm{ccc}>0.77)$ for all three datasets (Fig. 3 bottom row).

This observation motivated the second step of the analysis, the comparison of expression patterns between different cyanobacterial strains. To facilitate this comparison, the prediction of homologous genes in the cyanobacterial clade by Beck and colleagues [108] was used as starting point. We focused first on the set of genes with oscillating expression patterns in all datasets. A summary of the cellular functions represented in the core diurnal genome is shown in Fig. 4a (Additional file 2: Table S5), listing the frequencies of gene function category annotations of Cyanobase [71] for the respective Synechocystis 6803 genes. Three categories can be seen to dominate, i.e. Translation, Photosynthesis and Respiration, and Hypothetical. Most strikingly, 18 out of 64 genes annotated by Cyanobase [71] as "ribosomal protein" genes in Synechocystis 6803 fall into the core diurnal set, furthermore seven out of 27 genes annotated with "photosystem II". The remaining diurnally expressed genes are found interspersed across the metabolic network. While genes coding for parts of photosystem II, the RNA polymerase, or the ribosomal proteins can be expected to serve important roles in the adaptation to photic and aphotic phases, this analysis ascribes similar importance to other metabolic processes e.g. in the repair of UV-damaged Photosystem II centers (slr1390) [109], in the phosphate transport system (pstB2), pyrimidine and arginine biosynthesis (sll1498), or the glycolysis/gluconeogenesis via the fructose-bisphosphate aldolase (sll0018). Marker gene candidates for a working clock can be derived from the core diurnal genome. Importantly, less confidence can be placed in genes, which exhibit peak expression phases shortly past dawn, since these can either be clock regulated or simply induced by light. Two high confidence candidates are the light-independent protochlorophyllide reductase subunit $\mathrm{ChlB}$ and the fructose-bisphosphate aldolase $f b p$. Indeed, $f b p$ also shows circadian expression patterns in Chlamydomonas reinhardtii [110] and Arabidopsis thaliana where its late-night peaks may reflect the great importance of these aldolases in higher plants for the mobilization of plastidic starch [111]. Particularly in higher plants, the mobilization of starch, the conversion into sucrose, and its transport to other parts of the plant occur mainly at night.

The group of 15 "hypothetical protein" genes in the core diurnal genome constitutes an excellent candidate set for novel clock-driven genes in strains with a working core 
clock. Interestingly, several of these genes are implicated with cell division, such as the YlmG-related hypothetical gene ssl0353, which is required for proper distribution of nucleoids in Cyanobacteria and chloroplasts [112]. Similarly, the hypothetical protein $\operatorname{sir} 1577$ is suggested to function in the separation of chromosomes during cell division (Uniprot entry P74610). For the gene slr1847 (Uniprot entry P73057) a DNA binding capability is suggested, which could therefore regulate expression, aid nucleoid organization, or protect the DNA.

The core clock genes kaiA, kaiB, and kaiC are a good starting point for a detailed comparative expression analysis. Only the kaiB1 is significantly oscillating in all considered datasets. Interestingly, the kaiA gene features only very low amplitude expression oscillations and is arrhythmic in the vijayan09 Synechococcus 7942 dataset. The expression phases vary from dawn (Microcystis aeruginosa PCC 7806) to morning (Synechocystis 6803), over midday (stoeckel08 dataset of Cyanothece sp.
ATCC 51142), and dusk (ito09 dataset of Synechococcus 7942), into night (Anabaena sp. PCC 7120). The observed expression phases of kaiB1 are comparable to those of kaiA, but with significantly larger amplitude in Synechococcus 7942 datasets. The phase of the kaiB1 homolog in Prochlorococcus marinus MED4 peaks before dawn, comparable to Anabaena sp. PCC 7120. The kaiC1 expression phases and amplitudes match those of kaiB1, with the notable exception of Cyanothece sp. ATCC 51142 for which antiphasic late-night peaks are observed. In Prochlorococcus marinus MED4, kaiC1 peaks during the early night in contrast to the late night phase of kaiB1.

Many aspects agree well with previous knowledge. In Synechococcus 7942, the core clock genes kaiB and kaiC are arranged in the kaiBC operon resulting in similar expression patterns [113-115], while Cyanothece sp. ATCC 51142 features the kaiAB1C1 operon [116]. Interestingly, Cyanothece sp. ATCC 51142 features consistent anti-phasic expression of kaiB1 and kaiCl whereas the

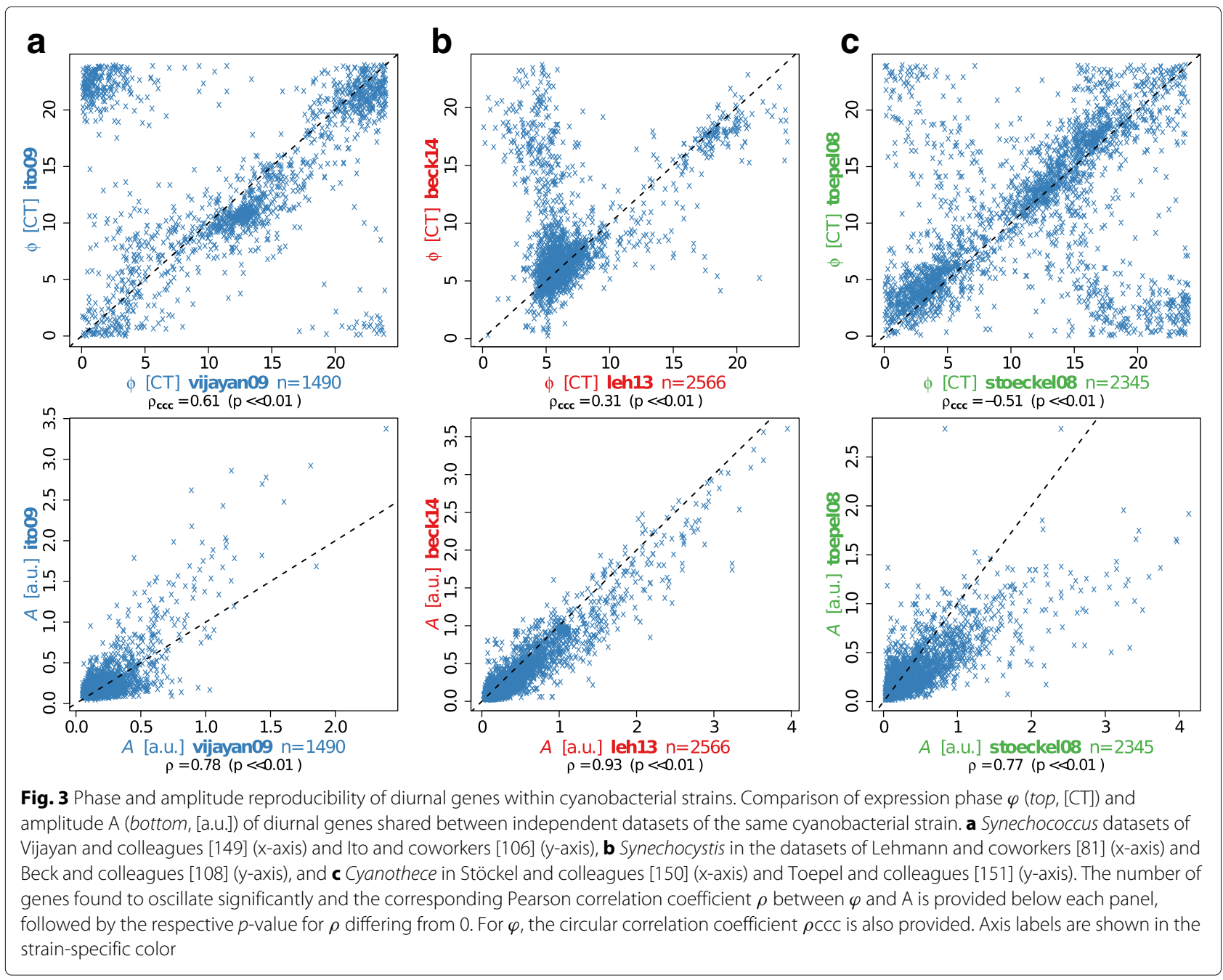



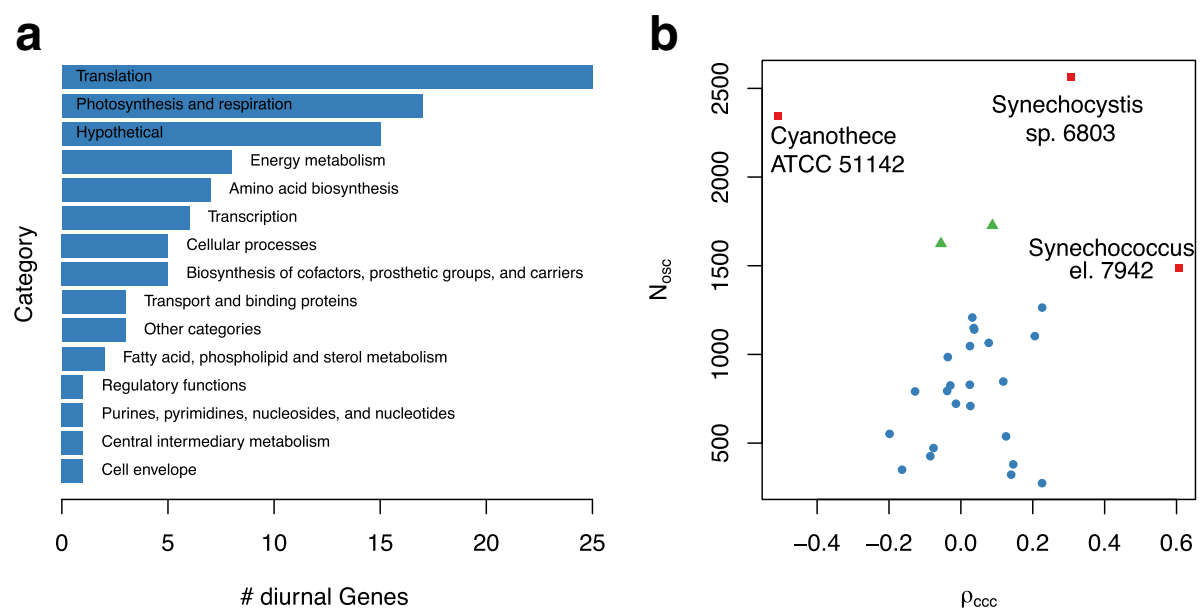

Fig. 4 The functional composition of the core diurnal transcriptome and expression phase similarity between all datasets. a The gene functional categories of the core diurnal gene set using the annotations provided by Cyanobase for Synechocystis 6803. $\mathbf{b}$ The comparison is carried out between all dataset combinations. Biological replicate experiments available for three cyanobacterial strains show a large number of shared oscillating genes as well as large correlation coefficients (red). The comparison of the stoeckel08 dataset (Cyanothece) with both Synechocystis datasets is marked in green. The remaining comparisons between various strains are shown in blue

remaining strains show co-expression, hinting at Cyanothece-specific post-transcriptional regulation of kaiB1 or kaiC1. Oscillations in the kaiA gene expression, as reported by Ishiura and colleagues, feature small expression amplitudes compared to $\mathrm{kaiB}$ and kaiC [117]. In fact, kaiA consistently falls below the threshold of 2fold expression change for the classification as circadian oscillator, which is commonly employed in microarray studies.

In the second step we generalized the detailed analysis of expression phases as presented for the core diurnal genome. We applied the circular correlation measure to all possible combinations of expression datasets. The resulting distribution reveals a clear separation between pairs of biological replicate datasets, featuring large numbers of shared oscillating genes and more extreme correlations (Fig. 4b red), and pairs of different cyanobacterial strains with fewer shared oscillating genes and much less extreme correlation coefficients (Fig. $4 \mathrm{~b}$ blue). The only exception to this separation is the Cyanothece sp. ATCC 51142 dataset stoeckel08 (Fig. 4b green), which shares many oscillating genes with both Synechocystis 6803 datasets (leh13, beck14). The corresponding correlation coefficients are, however, similarly small compared to other inter-strain pairs. The full set of pairwise phase comparisons, which underlay this analysis, are shown in Additional file 1: Figure S1. This result indicates that the diurnal peak expression phase is not preserved amongst homologous genes in the cyanobacterial clade but might instead be tuned according to the metabolic gene outfit and the environmental needs of the respective strain.

\section{Core circadian clock proteins, KaiA, KaiB, KaiC vary in number and length}

The previously observed diversity of circadian clocks within Cyanobacteria, and between other Bacteria and Archaea prompted further sequence analyses of the core clock proteins KaiA, KaiB and KaiC. Length comparisons gave rise to some new features of variations between the core factors of the circadian clock (Fig. 5). As described in the preceding, our BLAST analysis detected KaiA exclusively in Cyanobacteria. Interestingly, we could distinguish three subtypes of KaiA. While the sequence length of most KaiA is around 300 amino acids (AA) (Synechococcus 7942: 284 AA) some stains have shortend homologs with a length of roughly 200 and 100 AA, respectively (Fig. 5a, c). Truncated KaiA proteins are almost exclusively found in members of the order Nostocales. Similar results were reported previously by Dvornyk and colleagues, who also observed a higher degree of polymorphism for the kaiA gene in comparison to $\mathrm{kaiB}$ and $\mathrm{kaiC}[54,55]$. Multiple alignments of the KaiA proteins (Additional file 1: Figure S2) verified that the truncated KaiA proteins have a shortened N-terminal sequence, which functions in the complete protein as the amplitude amplifier [118]. However, all of these KaiA orthologs contain the C-terminal part important for clock oscillation [118].

Distribution of the KaiB protein length reveals two distinct groups. The KaiB homologs are either as long as the one from Synechococcus 7942 (102 AA) or about 250 AA in length (Fig. 5b, c). Microcoleus sp. PCC 7113 even has a KaiB with the length of 381 AA. KaiB homologs with query length are present in all four groups (Fig. 5b). 

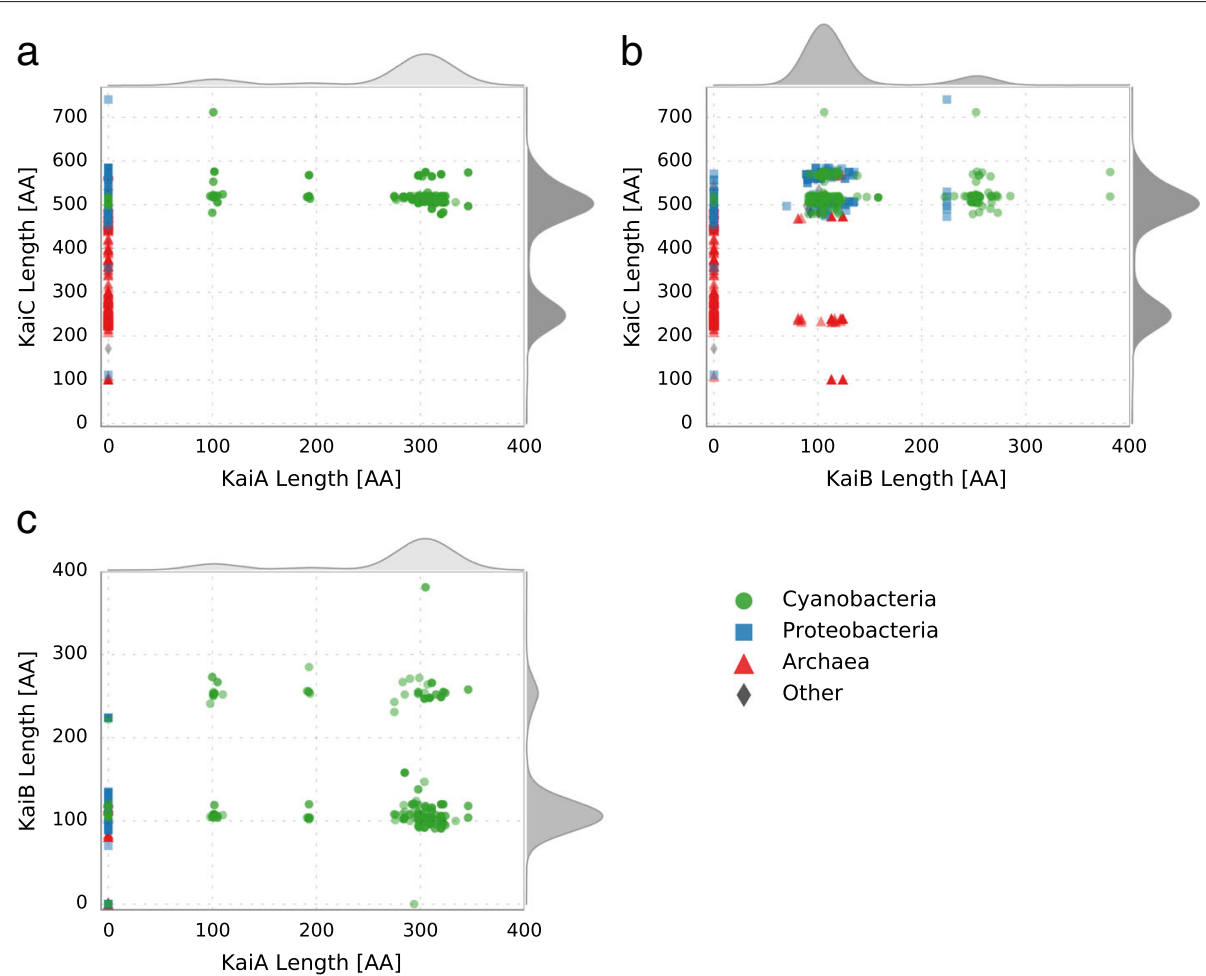

.5 Protein length distribution of the circadian clock factors from Synechococcus 7942 and its homologs. The four taxonomic main groups are highlighted: Cyanobacteria (Green), Proteobacteria (B/ue), Archaea (Red), Other (Grey). The curves outside of the plot represent the cumulative density distribution of the respective protein. a KaiC length distribution in dependency of the KaiA length. $\mathbf{b}$ KaiC length distribution in dependency of the KaiB length. $\mathbf{c}$ KaiB length distribution in dependency of the KaiA length

Elongated KaiB proteins are mainly present in Cyanobacteria in the subclass Oscillatoriophycideae and the order Nostocales, specifying findings of Dvornyk [55] (Fig. 5c). BLAST analyses using the KaiB homologs from Synechocystis 6803 revealed that elongated variants are most similar to KaiB1. Elongation via concatenation of two KaiB was ruled out by visually inspecting alignments with two artificially concatenated KaiB1. Instead the elongated KaiB1 have a $\sim 150$ AA N-terminal extension. BLAST searches of the $\mathrm{N}$-terminal region showed no homologous sequences in other organisms than Cyanobacteria, and no putative conserved domains could be identified. However, the N-terminal part is highly conserved within the Cyanobacteria having this KaiB variant. Interestingly, those Nostocales with an elongated KaiB1, also show a truncated KaiA.

The KaiC protein from Synechococcus 7942 is 519 AA in length and is build up by two domains, the $\mathrm{CI}$ and $\mathrm{CII}$ domain, which have a high similarity and are connected by a linker-domain $[119,120]$. The C-terminal CII domain of KaiC comprises the interaction sites with KaiA as well as the specific phosphorylation sites [29, 121-123]. KaiC homologs were detected with lengths varying between
101 AA and 741 AA (Fig. 5a, b). There are KaiC homologs in Archaea representing the whole observed length spectrum of $\mathrm{KaiC}$, whereas bacterial KaiCs are almost always about $500 \mathrm{AA}$ in length (Fig. 5a, b). Furthermore, in Bacteria and Archaea KaiB (and KaiA in Cyanobacteria) is only found when a 'full-length' KaiC is present. In these bacterial organisms the length of KaiC is almost constant, regardless of the length of KaiA and KaiB homologs (Fig. 5a, b). However, in KaiB-possessing Archaea additional shorter KaiC homologs are found (Fig. 5b).

Moreover, the length distribution of KaiC revealed a substantial amount of $\mathrm{KaiC}$ homologs with a length of circa $250 \mathrm{AA}$, which is approximately the length of one KaiC domain. This KaiC variant is mainly found in Archaea, but also in a few Bacteria. In these bacterial species no KaiB homolog could be identified. Shorter KaiC homologs do not contain the important phosphorylation sites for maintaining the oscillator function. Therefore, they might not restore the full functionality of the Synechococcus $7942 \mathrm{KaiC}$, but can rather answer questions about the evolution of KaiC [55]. Regarding the evolution of KaiC, two valid hypotheses exist, both of which state that KaiC arose from a shorter ancestral recA 
gene followed by a gene duplication and fusion. However, on the one hand Leipe and colleagues [124] hypothesize that an ancestral single-domain KaiC originated in Bacteria, was transferred into Archaea, where its two-domain version originated, and a second lateral transfer event introduced the double domain $\mathrm{KaiC}$ into Cyanobacteria. On the other hand Dvornyk and coworkers [55] argue in a follow up study that KaiC has to be of cyanobacterial origin. Given the amount of new genomic data further studies would help to unravel the evolutionary history of KaiC.

\section{Conserved motifs and activities in the cyanobacterial KaiC subgroups}

For KaiC2 homologs outside of the cyanobacterial phylum an involvement in stress response (Legionella pneumophila [125]) and adaptive growth under rhythmic conditions (Rhodopseudomonas palustris [59]) has been demonstrated. Both proteins display autophosphorylation and $\mathrm{KaiC} 2$ from Rhodopseudomonas palustris shows elevated ATPase activity [59]. Nevertheless, the function of cyanobacterial $\mathrm{KaiC} 2$ and $\mathrm{KaiC} 3$ homologs remains unclear. We already demonstrated that $\mathrm{KaiC} 2$ and KaiC3 from Synechocystis 6803 displays kinase activity, which is independent of KaiA, whereas KaiC1 behaved like its Synechococcus 7942 ortholog [57]. Those activities could also be predicted from the C-terminal amino acid sequences [57]. To test whether general features of the three KaiC subgroups can be predicted, multiple alignments of the cyanobacterial $\mathrm{KaiC} 1, \mathrm{KaiC} 2$ and KaiC3 sequences were constructed. A WebLogo analysis revealed that relevant motifs for phosphorylation and dephosphorylation in the CII domain are highly conserved. The ATP-binding Walker Motif A (P-loop in Fig. 6a, GXXXXGKT, [117, 126, 127]) is present in all three KaiC subgroups. Strikingly, the respective sequence of KaiC-7942 (GATGTGKT) shows almost no modifications in KaiC1 and KaiC3 proteins. Furthermore, catalytic glutamates (EE in Fig. 6a, [128, 129]), the R-finger contacting the $\gamma$-phosphate of ATP [130], and the truncated Walker motif B (Walker B in Fig. 6a, [26, 117, 127]), were found in all cyanobacterial KaiC subgroups. Notably, the arginine residue of the Synechococcus 7942 Walker B motif is not conserved in $\mathrm{KaiC} 2$ homologs. Serine and subsequent threonine are the dominant phosphorylation sites in $\mathrm{KaiC} 1$ and $\mathrm{KaiC} 3$ proteins, like S431 and T432 in KaiC7942 [121, 122], whereas KaiC2 homologs display two serine residues. In some KaiC3 homologs a tyrosin is present as second phosphorylation site. T426, which is important for dephosphorylation of KaiC-7942 [121, 128, 131, 132], is also highly conserved. Therefore, phosphorylation and dephosphorylation via autokinase [26], ATP synthase and ATPase activity $[27,128]$, respectively, are very likely for all cyanobacterial KaiC homologs. The same holds true for the N-terminal ATPase activity: We observed high conservation of the Walker motif A (P-loop in Fig. 6a), the catalytic glutamate residues (EE in Fig. 6a) and the $\mathrm{R}$-finger in the CI domains of all cyanobacterial KaiC subgroups. The presence of the R-linker in CI domains of $\mathrm{KaiC} 1$ and $\mathrm{KaiC} 3$ homologs indicate a structural coupling of the N-terminal CI and the C-terminal CII-domain as it was demonstrated for Thermosynechococcus KaiC [130], whereas KaiC2 homologs lack the R-linker in CI.

Crystal structures are available for KaiB1 homologs and KaiB2 from Legionella pneumophila [133-138]. Free KaiB1 crystallizes in a unique fold, but can adopt a fold switched state, which is a prerequisite for KaiC binding and is similar to the thioredoxin like fold observed in KaiB2 crystals [138, 139]. Residues K57, G88 and D90, which were suggested to regulate fold switching of KaiB-7942 [139], are the most prominent residues at the mentioned positions in KaiB1 proteins, but not highly conserved (Additional file 1: Figure S3). Hence, the question arises whether fold switching is conserved among KaiB1 proteins. Interestingly, G88 is highly conserved in KaiB2 proteins and hence free KaiB2 proteins may not necessarily favor the fold changed state. In KaiB3 all three residues show high conservation, which implies adoption of the ground state by the free protein and fold switching for putative KaiC binding.

KaiC homologs from the genus Prochlorococcus were classified as KaiC1 orthologs in our BLAST analysis (Fig. 1b). However, Prochlorococcus strains do not contain KaiA, and KaiC from Prochlorococcus marinus MED4 was demonstrated to phosphorylate independently of KaiA due to a modified A-loop sequence [88]. Therefore, we compared WebLogos of the A-loop sequence [28] for $\mathrm{KaiC} 1$ orthologs from Cyanobacteria with and without KaiA (Fig. 6a). The most obvious difference to KaiC1 proteins from cyanobacterial strains with KaiA is the presence of neutral glutamine in the second position, instead of a positively charged arginine. In $\mathrm{KaiC} 2$ and $\mathrm{KaiC} 3$ Weblogos motifs are even less conserved as already described for the Synechocystis 6803 representatives [57]. Interestingly, the KaiC3 WebLogo motif does not display any charged residue anymore. The absence of A-loop residues that are important to keep it in the buried state [28] indicate that the KaiA-independent phosphorylation is characteristic for KaiC2 and KaiC3 homologs. This is supported by the low conservation of the 438-444-loop and/or the 422loop, which are part of the interaction network that mediates inhibition of phosphorylation by the buried Aloops in Synechococcus 7942 [28, 120]. The high conservation of these loops in KaiA-lacking strains remains enigmatic.

KaiA from Thermosynechoccus BP-1 interacts with residues of the A-loop and further $\mathrm{C}$-terminal amino acids 


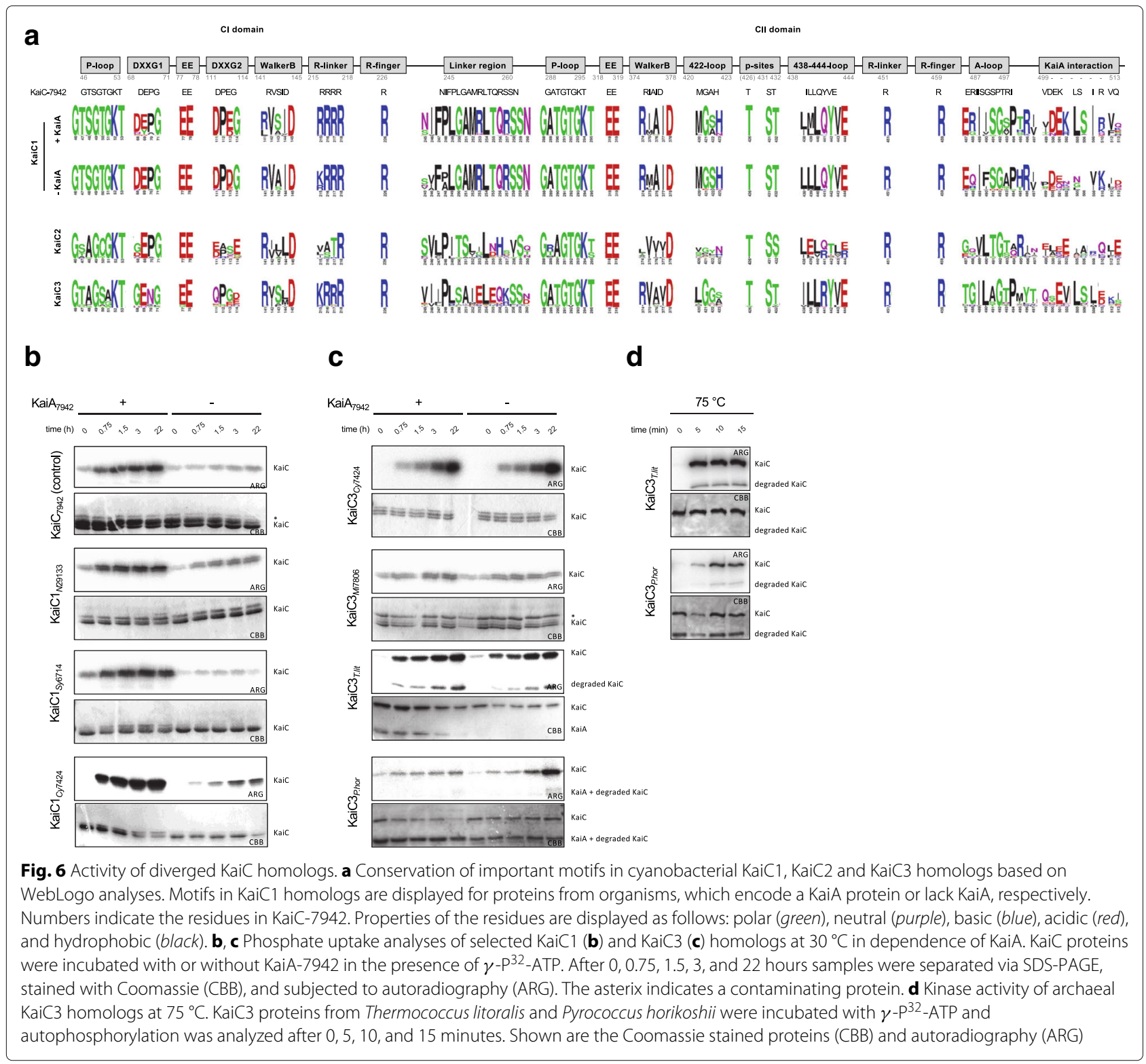

of Thermosynechococcus BP-1 KaiC [123, 140]. Direct comparison of KaiC1 proteins from strains with and without KaiA reveals that the lack of KaiA goes along with dramatically decreased conservation of the KaiA interacting residues (Fig. 6a). Due to the high variation of those residues in KaiC2 proteins, we predict that they do not interact with KaiA. However, several KaiA interacting amino acids are present or conservatively substituted in KaiC3 proteins. Hence, complex formation of KaiC3 and KaiA might be possible, but stimulation of kinase activity by KaiA is unlikely.

To test this hypothesis, phosphate uptake as an exemplary KaiC activity was analyzed for representative cyanobacterial KaiC1 and KaiC3 proteins by incubation with $\gamma$-P $\mathrm{P}^{32}$-ATP at $30{ }^{\circ} \mathrm{C}$ in the presence and absence of
Synechococcus 7942 KaiA (KaiA-7942). The well-studied KaiC from Synechococcus 7942 (KaiC-7942) served as control. As demonstrated in Fig. $6 \mathrm{~b}$ and $\mathrm{c}$ all recombinant KaiC proteins incorporated phosphate over time. The intrinsic kinase activity of KaiC1 homologs from Nostoc punctiforme ATCC 29413 (KaiC1-N294133), Synechocystis sp. PCC 6714 (KaiC1-Sy6714), and Cyanothece sp. PCC 7424 (KaiC1-Cy7424) was stimulated by KaiA-7942, similar to KaiC-7942 (Fig. 6b). As expected, KaiA had no effect on autophosphorylation of KaiC3 from Cyanothece sp. PCC 7424 (KaiC3-Cy7424) and Microcystis aeruginosa PCC 7806 (KaiC3-Mic7806, Fig. 6c). To extend the analysis to non-cyanobacterial proteins, KaiC3 from the hyperthermophilic Archaea Thermococcus litoralis (KaiC3-T.lit) and Pyrococcus horikoshii (KaiC3-P.hor), 
which show optimal growth at $85{ }^{\circ} \mathrm{C}$ and $98{ }^{\circ} \mathrm{C}$ [141, 142], were analyzed in a similar way. Again both recombinant KaiC3 proteins displayed phosphorylation at $30^{\circ} \mathrm{C}$, which was independent of KaiA-7942 (Fig. 6c). Incubation at $75^{\circ} \mathrm{C}$ indicated that the two archaeal KaiC3 homologs display kinase activity also at high growth temperatures (Fig. 6d). Hence, kinase activity of KaiC proteins seems to be well-conserved, independent of the growth conditions of the strains, they are originating from.

\section{Conclusion}

\section{A core module for circadian regulation}

Our analysis of 11,264 genomes clearly demonstrates that components of the Synechococcus 7942 circadian clock are present in various bacteria and archaea. However, the frequency of Kai-clock related proteins is highest in Cyanobacteria. In fact KaiA, Pex, LdpA, and CdpA are exclusive to organisms of this phylum. In other organisms, e.g. Rhodobacter sphaerodies, reduced KaiBC-based clock systems are likely able to drive circadian oscillations [96]. An even simpler system solely dependent on KaiC might enable diurnal rhythms in Haloferax volcanii [61], probably using the ATP/ADP ratio for clock entrainment. Predictions for KaiC activities based on sequence alignments and motif analyses were validated through biochemical experiments. We confirmed kinase activity for 'full-length' KaiC proteins composed of one $\mathrm{CI}$ and one CII domain, even in organism without kaiA or kaiB. KaiA from Synechococcus 7942 enhanced KaiC-phosphorylation only in strains naturally possessing a kaiA gene.

Our co-occurrence analysis hints to a conserved extension set for circadian regulation, which is present in Cyanobacteria with observed circadian behavior and absent in Cyanobacteria having a diurnal, hourglass-like lifestyle only (see also Fig. 7). A diurnal core set, which is important to enable an hourglass-like timing system that resets every day, might be composed of $\mathrm{KaiB}$, KaiC, LdpA, IrcA, SasA, RpaA, RpaB, and CpmA. However, our identified circadian core set, which potentially enables a selfsustained clock, additionally consists of KaiA, the two input factors CdpA, and PrkE as well as the input and output factor CikA, and the output factors LabA, and LalA.

The systematic comparison of microarray timeseries datasets indicates that the diurnal peak expression phase is not conserved amongst homologous genes in the cyanobacterial clade. Instead, the expression phase may be tuned according to the gene outfit and varying environmental needs. The analysis yielded a set of 95 genes in the core diurnal genome, which can be considered critical for the adaptation to day and night. Particularly, the subset of non-light induced genes are prime candidates for circadian clock marker genes. This set furthermore contains several hypothetical genes, which are interesting candidates for novel clock-driven genes.

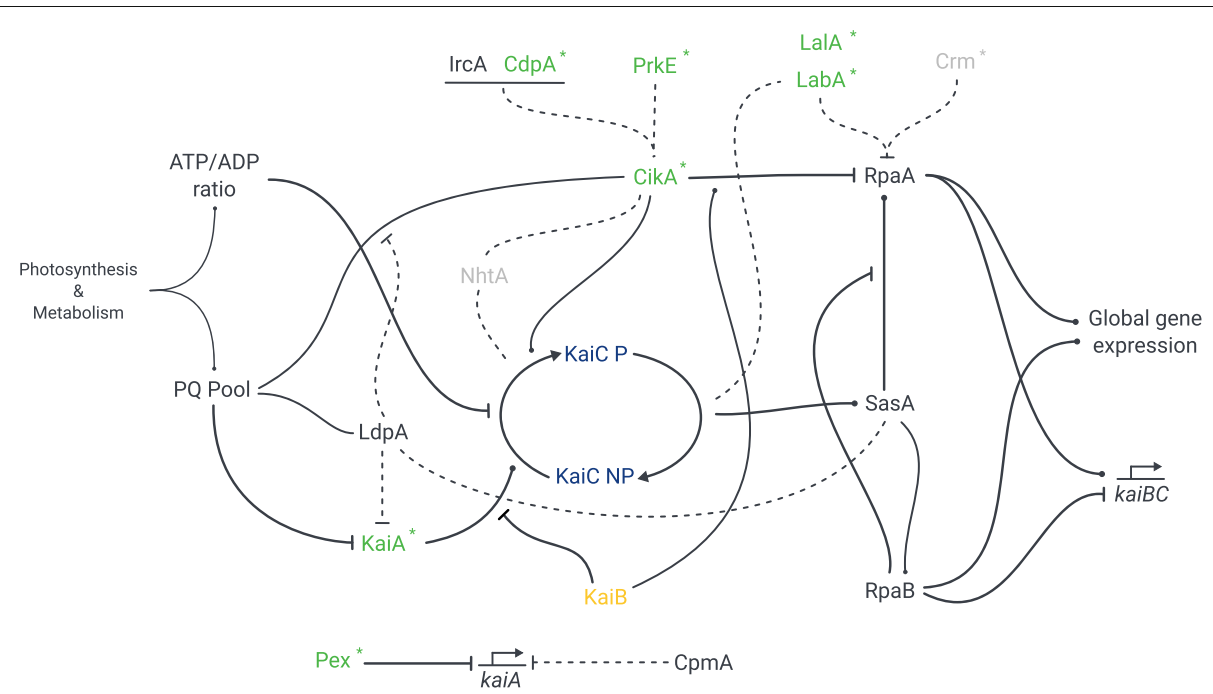

Fig. 7 Schematic overview of the circadian clock in Synechococcus 7942. Shown is the protein network in which the clock proteins are integrated based on $[20,36-53,103,139,152]$. The plastoquinone pool and ATP/ADP ratio serve as input signals to entrain the circadian clock with the environment and metabolic state of the cell. The signals are recognized by the input factors LdpA and CikA and the core clock factors KaiA and KaiC. Other input factors are Pex, and the CikA interaction partner NhtA, PrkE, IrcA, and CdpA. The stimulating effect of KaiA on KaiC is antagonized by KaiB. The output of the clock is comprised by SasA, CikA, RpaA, RpaB, LabA, LalA, Crm, and CpmA. SasA interacts with KaiC and further phosphorylates RpaA, whereas CikA acts as a phosphatase on RpaA. RpaA together with RpaB controls global gene expression as well as the expression of the kaiBC cluster. Solid lines represent established biochemical interactions whereas dashed lines represent inferred interactions. The seven most interconnected factors we found in our co-occurrence analysis are highlighted in green. Factors colored in light grey showed no co-occurrence in the Fisher's exact test and are present in $<90 \%$ of all observed systems. Asterisks $\left(^{*}\right)$ indicate the factors missing in Prochlorococcus 
The gained insights about the diversity within the composition of the components involved in the circadian protein clock as well as the diversity on the sequence level of the core factors call for further modification and simplification of the clock. The exponential increase of molecular tools for synthetic applications in recent years sets the stage for such ambitious projects. A future goal could be the reduction of complexity by removing as many factors as possible so that an integration of a circadian clock in synthetic and industrially valuable organisms becomes feasible.

\section{Additional files}

Additional file 1: Supplement 1. This file contains all supplementary figures and tables, except of Table S5. (PDF $9260 \mathrm{~kb}$ )

Additional file 2: Supplement 2. This file contains the data of Table 55 in a CSV file. (CSV $7 \mathrm{~kb})$

\section{Abbreviations}

AA: Amino Acid; Synechocystis 6803: Synechocystis sp. PCC 6803; Synechococcus 7942: Synechococcus elongatus PCC 7942

\section{Acknowledgement}

We thank Mariam Yazdanyar for help with protein expression and purification. We are very grateful to Elke Dittmann, Wolfgang Lockau, and Wolfgang R. Hess for providing genomic DNA of Microcystis aeruginosa PCC 7806 and Nostoc punctiforme ATCC 294133, Cyanothece PCC 7424, and Synechocystis PCC 6714, respectively.

\section{Funding}

This work was supported by DFG grants AX 84/1-3 and EXC 1028 to NMS, AW and IMA. ERC starting grant (Nr. 311523, Archaellum) for PC and SVA. RL was supported by the grant "CyanoGrowth" funded by the German Federal Ministry of Education and Research (reference: FKZ 0316192 to Ralf Steuer).

\section{Availability of data and materials}

The underlying raw and processed data of the BLAST experiments are available on figshare

(https://dx.doi.org/10.6084/m9.figshare.3823902.v3, https://dx.doi.org/10. $6084 / \mathrm{m} 9$. figshare.3823899.v3). The Python code is available in Jupyter Notebooks and archived on zenodo

(http://doi.org/10.5281/zenodo.229910). Protocols to the methods are uploaded to protocols.io. Everything is also noted in the respective method section for better clarification.

\section{Authors' contributions}

IMA and AW supervised the study. NMS, CB, and RL designed and NMS and RL carried out bioinformatic data analyses. AW designed and performed sequence analysis and in vitro experiments. AW and PC cloned, expressed, and purified recombinant proteins. NMS, RL, CB, AW, and IMA prepared the manuscript. PC and SVA contributed to the interpretation of the data and provided intellectual input. All authors read and approved the final manuscript.

\section{Competing interests}

The authors declare that they have no competing interests.

\section{Consent for publication}

Not applicable.

Ethics approval and consent to participate Not applicable.

\section{Publisher's Note}

Springer Nature remains neutral with regard to jurisdictional claims in published maps and institutional affiliations.

\section{Author details}

${ }^{1}$ Institute for Synthetic Microbiology, Cluster of Excellence on Plant Sciences (CEPLAS), Heinrich Heine University Duesseldorf, Universitaetsstrasse 1, 40225 Duesseldorf, Germany. ${ }^{2}$ Institute for Theoretical Biology, Humboldt University Berlin, Invalidenstrasse 43, 10115 Berlin, Germany. ${ }^{3}$ Molecular Biology of Archaea, Albert-Ludwigs-University Freiburg, Institute of Biology II,

Schaenzlestrasse 1, 79104 Freiburg, Germany.

Received: 13 March 2017 Accepted: 15 June 2017

Published online: 21 July 2017

\section{References}

1. Ouyang $Y$, Andersson CR, Kondo T, Golden SS, Johnson CH. Resonating circadian clocks enhance fitness in cyanobacteria. Proc Nat Acad Sci USA. 1998;95(15):8660-4.

2. Woelfle MA, Ouyang Y, Phanvijhitsiri $K$, Johnson $\mathrm{CH}$. The adaptive value of circadian clocks: an experimental assessment in cyanobacteria. Curr Biol. 2004;14(16):1481-6.

3. Simons MJ. The evolution of the cyanobacterial posttranslational clock from a primitive "phoscillator". J Biol Rhythms. 2009;24(3):175-82.

4. Ditty JL, Williams SB, Golden SS. A Cyanobacterial Circadian Timing Mechanism. Ann Rev Genet. 2003;37(1):513-43.

5. Johnson CH, Stewart PL, Egli M. The cyanobacterial circadian system: from biophysics to bioevolution. Ann Rev Biophys. 2011;40:143-67.

6. Golden SS, Canales SR. Cyanobacterial circadian clocks-timing is everything. Nat Rev Microbiol. 2003;1 (3):191-9.

7. Pittendrigh CS. Temporal organization: reflections of a Darwinian clock-watcher. Ann Rev Physiol. 1993;55:16-54.

8. Sweeney BM, Borgese MB. A circadian rhythm in cell division in a prokaryote, the cyanobacterium Synechococcus WH7803. J Phycol. 1989;25(1):183-6.

9. Mitsui A, Kumazawa S, Takahashi A, Ikemoto H, Cao S, Arai T. Strategy by which nitrogen-fixing unicellular cyanobacteria grow photoautotrophically. Nature. 1986;323(6090):720-2.

10. Huang TC, Chow TJ. New Type of N-2-Fixing Unicellular Cyanobacterium (Blue-Green-Alga). FEMS Microbiol Lett. 1986;36(1):109-10.

11. Grobbelaar N, Huang TC, Lin HY, Chow TJ. Dinitrogen-Fixing Endogenous Rhythm in Synechococcus Rf-1. FEMS Microbiol Lett. 1986;37(2):173-7.

12. Huang TC, Chow TJ. Characterization of the Rhythmic Nitrogen-Fixing Activity of Synechococcus sp. Rf- 1 at the Transcription Level. Curr Microbiol. 1990;20(1):23-6.

13. Tomita J, Nakajima M, Kondo T, Iwasaki H. No transcription-translation feedback in circadian rhythm of KaiC phosphorylation. Science. 2005;307(5707):251-4.

14. Nakajima M, Imai K, Ito H, Nishiwaki T, Murayama Y, Iwasaki H, Oyama T, Kondo T. Reconstitution of circadian oscillation of cyanobacterial KaiC phosphorylation in vitro. Science. 2005;308(5720):414-5.

15. Ditty JL, Canales SR, Anderson BE, Williams SB, Golden SS. Stability of the Synechococcus elongatus PCC 7942 circadian clock under directed anti-phase expression of the kai genes. Microbiology. 2005;151(8): 2605-613.

16. Edgar RS, Green EW, Zhao Y, van Ooijen G, Olmedo M, Qin X, Xu Y, Pan M, Valekunja UK, Feeney KA, Maywood ES, Hastings MH, Baliga NS, Merrow M, Millar AJ, Johnson CH, Kyriacou CP, O'Neill JS, Reddy AB. Peroxiredoxins are conserved markers of circadian rhythms. Nature. 2012;485(7399):459-64.

17. O'Neill JS, Reddy AB. Circadian clocks in human red blood cells. Nature. 2011;469(7331):498-503.

18. O'Neill JS, van Ooijen G, Dixon LE, Troein C, Corellou F, Bouget FY, Reddy AB, Millar AJ. Circadian rhythms persist without transcription in a eukaryote. Nature. 2011;469(7331):554-8.

19. McClung CR. Circadian Rhythms in Plants. Ann Rev Plant Physiol and Plant Mol Biol. 2001;52:139-62.

20. Ivleva NB, Bramlett MR, Lindahl PA, Golden SS. LdpA: a component of the circadian clock senses redox state of the cell. EMBO J. 2005;24(6): 1202-1210. 
21. Pattanayak GK, Rust MJ. The Cyanobacterial Clock and Metabolism. Curr Opin Microbiol. 2014;18:90-5.

22. Kim YI, Vinyard DJ, Ananyev GM, Dismukes GC, Golden SS. Oxidized quinones signal onset of darkness directly to the cyanobacterial circadian oscillator. Proc Nat Acad Sci USA. 2012;109(44):17765-9.

23. Hihara Y, Sonoike K, Kanehisa M, Ikeuchi M. DNA microarray analysis of redox-responsive genes in the genome of the cyanobacterium Synechocystis sp. strain PCC 6803. J Bacteriol. 2003;185(5):1719-25.

24. Hayashi F, Suzuki H, Iwase R, Uzumaki T, Miyake A, Shen JR, Imada K, Furukawa Y, Yonekura K, Namba K, Ishiura M. ATP-induced hexameric ring structure of the cyanobacterial circadian clock protein KaiC. Genes Cells. 2003;8(3):287-96.

25. Mori T, Saveliev SV, Xu Y, Stafford WF, Cox MM, Inman RB, Johnson $\mathrm{CH}$. Circadian clock protein KaiC forms ATP-dependent hexameric rings and binds DNA. Proc Nat Acad Sci USA. 2002;99(26):17203-8.

26. Nishiwaki T, Iwasaki H, Ishiura M, Kondo T. Nucleotide binding and autophosphorylation of the clock protein KaiC as a circadian timing process of cyanobacteria,. Proc Nat Acad Sci USA. 2000;97(1):495-9.

27. Nishiwaki T, Kondo T. Circadian autodephosphorylation of cyanobacterial clock protein KaiC occurs via formation of ATP as intermediate. J Biol Chem. 2012;287(22):18030-5.

28. Kim YI, Dong G, Carruthers JC, Golden SS, LiWang A. The day/night switch in KaiC, a central oscillator component of the circadian clock of cyanobacteria. Proc Nat Acad Sci USA. 2008;105(35):12825-30.

29. Pattanayek R, Williams DR, Pattanayek S, Xu Y, Mori T, Johnson CH, Stewart PL, Egli M. Analysis of KaiA-KaiC protein interactions in the cyanobacterial circadian clock using hybrid structural methods. EMBO J. 2006;25(9):2017-8

30. Rust MJ, Golden SS, O'Shea EK. Light-driven changes in energy metabolism directly entrain the cyanobacterial circadian oscillator Science. 2011:331(6014):220-3.

31. Kitayama Y, Iwasaki H, Nishiwaki T, Kondo T. KaiB functions as an attenuator of KaiC phosphorylation in the cyanobacterial circadian clock system. EMBO J. 2003;22(9):2127-34.

32. Tseng R, Goularte NF, Chavan A, Luu J, Cohen SE, Chang YG, Heisler J, Li S, Michael AK, Tripathi S, Golden SS, LiWang A, Partch CL. Structural basis of the day-night transition in a bacterial circadian clock. Science. 2017:355(6330):1174-80.

33. Snijder J, Schuller JM, Wiegard A, Lössl P, Schmelling N, Axmann IM, Plitzko JM, Förster F, Heck AJR. Structures of the cyanobacterial circadian oscillator frozen in a fully assembled state. Science. 2017;355(6330):1181-4.

34. Williams SB, Vakonakis I, Golden SS, LiWang AC. Structure and function from the circadian clock protein KaiA of Synechococcus elongatus: a potential clock input mechanism. Proc Nat Acad Sci USA. 2002;99(24): 15357-62

35. Wood TL, Bridwell-Rabb J, Kim Yl, Gao T, Chang YG, LiWang A Barondeau DP, Golden SS. The KaiA protein of the cyanobacterial circadian oscillator is modulated by a redox-active cofactor. Proc Nat Acad Sci USA. 2010;107(13):5804-9.

36. Kutsuna S, Kondo T, Aoki S. A Period-Extender Gene, pex, That Extends the Period of the Circadian Clock in the Cyanobacterium Synechococcus sp. Strain PCC 7942. J Bacteriol. 1998;180(8):2167-74.

37. Kutsuna S, Kondo T, Ikegami H, Uzumaki T, Katayama M, Ishiura M. The circadian clock-related gene pex regulates a negative cis element in the kaiA promoter region. J Bacteriol. 2007;189(21):7690-6.

38. Ivleva NB, Gao T, LiWang AC, Golden SS. Quinone sensing by the circadian input kinase of the cyanobacterial circadian clock. Proc Nat Acad Sci USA. 2006;103(46):17468-17473.

39. Schmitz O, Katayama M, Williams SB, Kondo T, Golden SS. CikA, a bacteriophytochrome that resets the cyanobacterial circadian clock, Science. 2000;289(5480):765-8.

40. Mackey SR, Choi JS, Kitayama Y, Iwasaki H, Dong G, Golden SS. Proteins found in a CikA interaction assay link the circadian clock, metabolism, and cell division in Synechococcus elongatus. J Bacteriol. 2008;190(10):3738-46.

41. Iwasaki H, Williams SB, Kitayama Y, Ishiura M, Golden SS, Kondo T. A KaiC-interacting sensory histidine kinase, SasA, necessary to sustain robust circadian oscillation in cyanobacteria. Cell. 2000;101(2):223-33.

42. Smith RM, Williams SB. Circadian rhythms in gene transcription imparted by chromosome compaction in the cyanobacterium Synechococcus elongatus. Proc Nat Acad Sci USA. 2006;103(22):8564-9.
43. Takai N, Nakajima M, Oyama $T$, Kito R, Sugita C, Sugita M, Kondo T, Iwasaki H. A KaiC-associating SasA-RpaA two-component regulatory system as a major circadian timing mediator in cyanobacteria. Proc Nat Acad Sci USA. 2006;103(32):12109-12114.

44. Hanaoka M, Takai N, Hosokawa N, Fujiwara M, Akimoto Y, Kobori N, Iwasaki $\mathrm{H}$, Kondo $\mathrm{T}$, Tanaka K. RpaB, another response regulator operating circadian clock-dependent transcriptional regulation in Synechococcus elongatus PCC 7942. J Biol Chem. 2012;287(31):26321-7.

45. Markson JS, Piechura JR, Puszynska AM, O'Shea EK. Circadian control of global gene expression by the cyanobacterial master regulator RpaA. Cell. 2013:155(6):1396-408.

46. Gutu A, O'Shea EK. Two Antagonistic Clock-Regulated Histidine Kinases Time the Activation of Circadian Gene Expression. Mol Cell. 2013;50(2): 288-94

47. Taniguchi $Y$, Nishikawa T, Kondo T, Oyama T. Overexpression of IalA, a paralog of $l a b A$, is capable of affecting both circadian gene expression and cell growth in the cyanobacterium Synechococcus elongatus PCC 7942. FEBS Lett. 2012;586(6):753-9.

48. Boyd JS, Bordowitz JR, Bree AC, Golden SS. An allele of the crm gene blocks cyanobacterial circadian rhythms. Proc Nat Acad Sci USA. 2013;110(34):13950-5.

49. Espinosa J, Boyd JS, Cantos R, Salinas P, Golden SS, Contreras A. Cross-talk and regulatory interactions between the essential response regulator RpaB and cyanobacterial circadian clock output. Proc Nat Acad Sci. 2015;112(7):2198-2203. doi:10.1073/pnas.1424632112.

50. Moronta-Barrios F, Espinosa J, Contreras A. In vivo features of signal transduction by the essential response regulator RpaB from Synechococcus elongatus PCC 7942. Microbiology. 2012;158(5):1229-37.

51. Katayama M, Tsinoremas NF, Kondo T, Golden SS. cpmA, a gene involved in an output pathway of the cyanobacterial circadian system. J Bacteriol. 1999;181(11):3516-24.

52. Taniguchi Y, Katayama M, Ito R, Takai N, Kondo T, Oyama T. IabA: a novel gene required for negative feedback regulation of the cyanobacterial circadian clock protein KaiC. Genes Dev. 2007;21(1): 60-70. doi:10.1101/gad.1488107.

53. Moronta-Barrios F, Espinosa J, Contreras A. Negative control of cell size in the cyanobacterium Synechococcus elongatus PCC 7942 by the essential response regulator RpaB. FEBS Lett. 2013;587(5):504-9.

54. Dvornyk V. The Circadian Clock Gear in Cyanobacteria: Assembled by Evolution In: Ditty JL, Mackey KR, Johnson CH, editors. Berlin Heidelberg: Springer; 2009. p. 241-58. Chap. 14.

55. Dvornyk V, Vinogradova O, Nevo E. Origin and evolution of circadian clock genes in prokaryotes. Proc Nat Acad Sci USA. 2003;100(5): 2495-500.

56. Axmann IM, Hertel S, Wiegard A, Dörrich AK, Wilde A. Diversity of KaiC-based timing systems in marine Cyanobacteria. Marine Genomics. 2014;14:3-16.

57. Wiegard A, Dörrich AK, Deinzer HT, Beck C, Wilde A, Holtzendorff J, Axmann IM. Biochemical analysis of three putative KaiC clock proteins from Synechocystis sp. PCC 6803 suggests their functional divergence. Microbiology. 2013;159(5):948-58.

58. Aoki S, Onai K. Circadian clocks of Synechocystis sp. strain PCC 6803, Thermosynechococcus elongatus, Prochlorococcus spp., Trichodesmium spp. and other species In: Ditty JL, Mackey SR, Johnson CH, editors. Bacterial Circadian Programs. Chapter 15. Berlin Heidelberg: Springer Berlin Heidelberg; 2009. p. 259-282.

59. Ma P, Mori T, Zhao C, Thiel T, Johnson CH. Evolution of KaiC-Dependent Timekeepers: A Proto-circadian Timing Mechanism Confers Adaptive Fitness in the Purple Bacterium Rhodopseudomonas palustris. PLoS Genet. 2016;12(3):1005922.

60. Kang HJ, Kubota K, Ming H, Miyazono K, Tanokura M. Crystal structure of KaiC-like protein PH0186 from hyperthermophilic archaea Pyrococcus horikoshii OT3. Proteins. 2009;75(4):1035-9.

61. Maniscalco M, Nannen J, Sodi V, Silver G, Lowrey PL, Bidle KA. Light-dependent expression of four cryptic archaeal circadian gene homologs. Front Microbiol. 2014;5:79.

62. Baca I, Sprockett D, Dvornyk V. Circadian input kinases and their homologs in cyanobacteria: Evolutionary constraints versus architectura diversification. J Mol Evol. 2010;70(5):453-65.

63. Dvornyk V, Deng HW, Nevo E. Structure and molecular phylogeny of sasA genes in cyanobacteria: Insights into evolution of the prokaryotic circadian system. Mol Biol Evol. 2004;21(8):1468-76. 
64. Cock PJ, Antao T, Chang JT, Chapman BA, Cox CJ, Dalke A, Friedberg I, Hamelryck T, Kauff F, Wilczynski B, de Hoon MJ. Biopython: freely available Python tools for computational molecular biology and bioinformatics. Bioinformatics. 2009;25(11):1422-3.

65. Pérez F, Granger E. IPython: A System for Interactive Scientific Computing, Computing in Science and Engineering. Comput Sci Eng. 2007;9(3):21-9.

66. van der Walt S, Colbert SC, Varoquaux G. The NumPy Array: A Structure for Efficient Numerical Computation. Comput Sci Eng. 2011;13(2):22-30.

67. Hunter JD. Matplotlib: A 2D Graphics Environment. Comput Sci Eng. 2007;9(3):90-5

68. McKinney W. Data structures for statistical computing in Python In: van der Walt S, Millman J, editors. Proceedings of the 9th Python in Science Conference; 2010. p. 51-56. http://conference.scipy.org/ proceedings/scipy2010/mckinney.html.

69. Schmelling NM. Code from: Minimal Tool Set for a Prokaryotic Circadian Clock. zenodo Repository. 2017. http://doi.org/10.5281/zenodo.229910.

70. Benson DA, Karsch-Mizrachi I, Lipman DJ, Ostell J, Wheeler DL. GenBank. Nucleic Acids Res. 2008;36:25-30.

71. Nakao M, Okamoto S, Kohara M, Fujishiro T, Fujisawa T, Sato S, Tabata S, Kaneko T, Nakamura Y. CyanoBase: the cyanobacteria genome database update 2010. Nucleic Acids Res. 2010;38:379-81.

72. Altschul SF, Madden TL, Schaffer AA, Zhang J, Zhang Z, Miller W, Lipman DJ. Gapped BLAST and PSI-BLAST: a new generation of protein database search programs. Nucleic Acids Res. 1997;25(17):3389-402.

73. Schmelling NM. Raw Data from: Minimal Tool Set for a Prokaryotic Circadian Clock. figshare Repository. 2017. https://doi.org/10.6084/m9. figshare.3823902.v3.

74. Schmelling NM. Processed Data from: Minimal Tool Set for a Prokaryotic Circadian Clock. figshare Repository. 2017. https://doi.org/10.6084/m9. figshare.3823899.v3.

75. Fisher RA. On the Interpretation of $\chi^{2}$ from Contingency Tables, and the Calculation of P. J R Stat Soc. 1922;85(1):87-94

76. Benjamini Y, Hochberg Y. Controlling the False Discovery Rate: A Practical and Powerful Approach to Multiple Testing. J R Stat Soc. Series B (Methodological). 1995;57(1):289-300

77. Labiosa RG, Arrigo KR, Tu CJ, Bhaya D, Bay S, Grossman AR, Shrager J. Examination of diel changes in global transcript accumulation in Synechocystis (Cyanobacteria). J Phycol. 2006;42(3):622-36.

78. Kucho Kl, Okamoto K, Tsuchiya Y, Nomura S, Nango M, Kanehisa M, Ishiura M. Global analysis of circadian expression in the cyanobacterium Synechocystis sp. strain PCC 6803. J Bacteriol. 2005;187(6):2190-9.

79. Toepel JR, McDermott JE, Summerfield TC, Sherman LA. Transcriptional analysis of the unicellular, diazotrophic cyanobacterium Cyanothece sp. 51142 grown under short day/night cycles. J Phycol. 2009;45(3):610-20.

80. Kushige $H$, Kugenuma $H$, Matsuoka M, Ehira S, Ohmori M, Iwasaki H. Genome-wide and heterocyst-specific circadian gene expression in the filamentous Cyanobacterium Anabaena sp. strain PCC 7120. J Bacteriol. 2013;195(6):1276-84

81. Lehmann R, Machné R, Georg J, Benary M, Axmann IM, Steuer R. How cyanobacteria pose new problems to old methods: challenges in microarray time series analysis. BMC Bioinforma. 2013;14:133.

82. Yang Q, Pando BF, Dong G, Golden SS, van Oudenaarden A. Circadian gating of the cell cycle revealed in single cyanobacterial cells. Science. 2010;327(5972):1522-6.

83. Westermark PO, Herzel H. Mechanism for $12 \mathrm{hr}$ rhythm generation by the circadian clock. Cell Rep. 2013;3(4):1228-38.

84. Sievers F, Wilm A, Dineen D, Gibson TJ, Karplus K, Li W, Lopez R, McWilliam H, Remmert M, Soding J, Thompson JD, Higgins DG. Fast, scalable generation of high-quality protein multiple sequence alignments using Clustal Omega. Mol Syst Biol. 2011;7:539.

85. Waterhouse AM, Procter JB, Martin DMA, Clamp M, Barton GJ. Jalview Version 2-a multiple sequence alignment editor and analysis workbench. Bioinformatics. 2009;25(9):1189-91.

86. Crooks GE, Hon G, Chandonia JM, Brenner SE. WebLogo: A Sequence Logo Generator. Genome Res. 2004;14(6):1188-90.

87. Laemmli UK. Cleavage of structural proteins during the assembly of the head of bacteriophage T4. Nature. 1970;227(5259):680-5.

88. Axmann IM, Dühring U, Seeliger L, Arnold A, Vanselow JT, Kramer A, Wilde A. Biochemical evidence for a timing mechanism in Prochlorococcus. J Bacteriol. 2009;191(17):5342-7.
89. Loza-Correa M, Gomez-Valero L, Buchrieser C. Circadian clock proteins in prokaryotes: hidden rhythms? Front Microbiol. 2010;1:130.

90. Nakayama T, Kamikawa R, Tanifuji G, Kashiyama Y, Ohkouchi N, Archibald JM, Inagaki Y. Complete genome of a nonphotosynthetic cyanobacterium in a diatom reveals recent adaptations to an intracellular lifestyle. Proc Nat Acad Sci USA. 2014;111(31):11407-11412.

91. Nakamura Y, Kaneko T, Sato S, Mimuro M, Miyashita H, Tsuchiya T, Sasamoto S, Watanabe A, Kawashima K, Kishida Y, Kiyokawa C, Kohara M, Matsumoto M, Matsuno A, Nakazaki N, Shimpo S, Takeuchi C, Yamada M, Tabata S. Complete Genome Structure of Gloeobacter violaceus PCC 7421, a Cyanobacterium that Lacks Thylakoids. DNA Res. 2003;10(4):137-45.

92. Holtzendorff J, Partensky F, Mella D, Lennon JF, Hess WR, Garczarek L. Genome streamlining results in loss of robustness of the circadian clock in the marine cyanobacterium Prochlorococcus marinus PCC 9511. J Biol Rhythms. 2008;23(3):187-99.

93. Shultzaberger RK, Boyd JS, Katsuki T, Golden SS, Greenspan RJ. Single mutations in sasA enable a simpler $\triangle$ cikA gene network architecture with equivalent circadian properties. Proc Nat Acad Sci USA. 2014;111(47):5069-75.

94. Dvornyk V. Molecular evolution of IdpA, a gene mediating the circadian input signal in cyanobacteria. J Mol Evol. 2005;60(1):105-12.

95. Katayama M, Kondo T, Xiong J, Golden SS. IdpA Encodes an Iron-Sulfur Protein Involved in Light-Dependent Modulation of the Circadian Period in the Cyanobacterium Synechococcus elongatus PCC 7942. J Bacteriol. 2003;185(4):1415-22.

96. Min H, Guo H, Xiong J. Rhythmic gene expression in a purple photosynthetic bacterium, Rhodobacter sphaeroides. FEBS Lett. 2005;579(3):808-12.

97. Nishiwaki-Ohkawa T, Kitayama Y, Ochiai E, Kondo T. Exchange of ADP with ATP in the CII ATPase domain promotes autophosphorylation of cyanobacterial clock protein KaiC. Proc Nat Acad Sci USA. 2014;111(12): 4455-60.

98. Dong G, Yang Q, Wang Q, Kim Yl, Wood TL, Osteryoung KW, van Oudenaarden A, Golden SS. Elevated ATPase activity of KaiC applies a circadian checkpoint on cell division in Synechococcus elongatus. Cell. 2010;140(4):529-39.

99. Taniguchi Y, Takai N, Katayama M, Kondo T, Oyama T. Three major output pathways from the KaiABC-based oscillator cooperate to generate robust circadian kaiBC expression in cyanobacteria. Proc Nat Acad Sci USA. 2010;107(7):3263-3268. doi:10.1073/pnas.0909924107.

100. Vakonakis I, Klewer DA, Williams SB, Golden SS, LiWang AC. Structure of the N-terminal Domain of the Circadian Clock-associated Histidine Kinase SasA. J Mol Biol. 2004;342(1):9-17.

101. Shi Y, Tyson GW, Eppley JM, DeLong EF. Integrated metatranscriptomic and metagenomic analyses of stratified microbial assemblages in the open ocean. ISME J. 2011;5(6):999-1013.

102. Wilde A, Hihara Y. Transcriptional and posttranscriptional regulation of cyanobacterial photosynthesis. Biochimica et Biophysica Acta (BBA) Bioenergetics. 2016;1857(3):296-308. http://dx.doi.org/10.1016/j.bbabio. 2015.11.002.

103. Shultzaberger RK, Boyd JS, Diamonad S, Greenspan RJ, Golden SS. Giving Time Purpose: The Synechococcus elongatus Clock in a Broader Network Context. Ann Rev Genet. 2015;49(1):485-505.

104. Liu Y, Tsinoremas NF, Johnson CH, Lebedeva NV, Golden SS, Ishiura M, Kondo T. Circadian orchestration of gene expression in cyanobacteria. Genes Dev. 1995;9(12):1469-78.

105. Woelfle MA, Johnson CH. No Promoter Left Behind: Global Circadian Gene Expression in Cyanobacteria. J Biol Rhythms. 2006;21(6):419-31.

106. Ito H, Mutsuda M, Murayama Y, Tomita J, Hosokawa N, Terauchi K, Sugita C, Sugita M, Kondo T, Iwasaki H. Cyanobacterial daily life with Kai-based circadian and diurnal genome-wide transcriptional control in Synechococcus elongatus. Proc Nat Acad Sci USA. 2009;106(33):14168-73.

107. Jammalamadaka SR, SenGupta A, Vol. 5. Topics in Circular Statistics: World Scientific. 2001. http://www.worldscientific.com/worldscibooks/ $10.1142 / 4031$.

108. Beck C, Hertel S, Rediger A, Lehmann R, Wiegard A, Kölsch A, Heilmann B, Georg J, Hess WR, Axmann IM. Daily expression pattern of protein-encoding genes and small non coding RNAs in Synechocystis sp. strain PCC 6803. Appl Environ Microbiol. 2014;80(17):5195-206. 
109. Cheregi O, Sicora C, Kos PB, Nixon PJ, Vass I. The FtsH protease is required for the repair of Photosystem II in the cyanbacterium Synechocystis sp. PCC 6803 damaged UV-B radiation. BMC Plant Biol. 2005;5(1):1-2.

110. Jacobshagen S, Whetstine JR, Boling JM. Many but not all genes in Chlamydomonas reinhardtii are regulated by the Circadian Clock. Plant Biol. 2001;3(6):592-7

111. Harmer SL, Hogenesch JB, Straume M, Chang HS, Han B, Zhu T, Wang X, Kreps JA, Kay SA. Orchestrated Transcription of Key Pathways in Arabidopsis by the Circadian Clock. Science. 2000;290(5499):2110-3.

112. Kabeya Y, Nakanishi H, Suzuki K, Ichikawa T, Kondou Y, Matsui M, Miyagishima SY. The YImG protein has a conserved function related to the distribution of nucleoids in chloroplasts and cyanobacteria. BMC Plant Biol. 2010;10(1):1-13.

113. Imai K, Nishiwaki T, Kondo T, Iwasaki H. Circadian rhythms in the synthesis and degradation of a master clock protein KaiC in cyanobacteria. J Biol Chem. 2004;279(35):36534-9.

114. Iwasaki $H$, Nishiwaki T, Kitayama $Y$, Nakajima M, Kondo T. KaiA-stimulated KaiC phosphorylation in circadian timing loops in cyanobacteria. Proc Nat Acad Sci USA. 2002;99(24):15788-93.

115. Zwicker D, Lubensky DK, Ten Wolde PR. Robust circadian clocks from coupled protein-modification and transcription-translation cycles. Proc Nat Acad Sci USA. 2010;107(52):22540-5.

116. Memon D, Singh AK, Pakrasi HB, Wangikar PP. A global analysis of adaptive evolution of operons in cyanobacteria. Antonie van Leeuwenhoek. 2013;103(2):331-46.

117. Ishiura M, Kutsuna S, Aoki S, Iwasaki H, Andersson CR, Tanabe A, Golden SS, Johnson CH, Kondo T. Expression of a gene cluster kaiABC as a circadian feedback process in cyanobacteria. Science. 1998;281(5382):1519-23

118. Uzumaki T, Fujita M, Nakatsu T, Hayashi F, Shibata H, Itoh N, Kato H, Ishiura M. Crystal structure of the C-terminal clock-oscillator domain of the cyanobacterial KaiA protein. Nat Struct and Mol Biol. 2004;11(7): 623-31.

119. Iwasaki H, Taniguchi $Y$, Ishiura M, Kondo T. Physical interactions among circadian clock proteins KaiA, KaiB and KaiC in cyanobacteria. EMBO J. 1999;18(5):1137-45.

120. Egli M, Pattanayek R, Sheehan JH, Xu Y, Mori T, Smith JA, Johnson CH. Loop-loop interactions regulate KaiA-stimulated KaiC phosphorylation in the cyanobacterial KaiABC circadian clock. Biochemistry. 2013;52(7): 1208-20.

121. Xu Y, Mori T, Pattanayek R, Pattanayek S, Egli M, Johnson CH. Identification of key phosphorylation sites in the circadian clock protein KaiC by crystallographic and mutagenetic analyses. Proc Nat Acad Sci USA. 2004;101(38):13933-8.

122. Nishiwaki T, Satomi $Y$, Nakajima M, Lee C, Kiyohara R, Kageyama $H$, Kitayama Y, Temamoto M, Yamaguchi A, Hijikata A, Go M, Iwasaki H, Takao T, Kondo T. Role of KaiC phosphorylation in the circadian clock system of Synechococcus elongatus PCC 7942. Proc Nat Acad Sci USA. 2004;101(38):13927-32.

123. Vakonakis I, LiWang AC. Structure of the C-terminal domain of the clock protein KaiA in complex with a KaiC-derived peptide: implications for KaiC regulation. Proc Nat Acad Sci USA. 2004;101(30):10925-30.

124. Leipe DD, Aravind L, Grishin NV, Koonin EV. The Bacterial Replicative Helicase DnaB Evolved from a RecA Duplication. Genome Res. 2000;10(1):5-16.

125. Loza-Correa M, Sahr T, Rolando M, Daniels C, Petit P, Skarina T, Gomez Valero L, Dervins-Ravault D, Honoré N, Savchenko A Buchrieser C. The Legionella pneumophila kai operon is implicated in stress response and confers fitness in competitive environments. Environ Microbiol. 2014;16(2):359-81.

126. Pattanayek R, Wang J, Mori T, Xu Y, Johnson CH, Egli M. Visualizing a circadian clock protein: crystal structure of KaiC and functional insights. Mol Cell. 2004;15(3):375-88.

127. Walker JE, Saraste M, Runswick MJ, Gay NJ. Distantly related sequences in the alpha- and beta-subunits of ATP synthase, myosin, kinases and other ATP-requiring enzymes and a common nucleotide binding fold. EMBO J. 1982;1(8):945-51.

128. Egli M, Mori T, Pattanayek R, Xu Y, Qin X, Johnson CH. Dephosphorylation of the core clock protein KaiC in the cyanobacterial KaiABC circadian oscillator proceeds via an ATP synthase mechanism. Biochemistry. 2012;51(8):1547-58.
129. Kitayama Y, Nishiwaki-Ohkawa T, Sugisawa Y, Kondo T. KaiC intersubunit communication facilitates robustness of circadian rhythms in cyanobacteria. Nat Commun. 2013;4:2897.

130. Pattanayek R, Xu Y, Lamichhane A, Johnson CH, Egli M. An arginine tetrad as mediator of input-dependent and input-independent ATPases in the clock protein KaiC. Acta Crystallographica Section D: Biol Crystallograph. 2014;70(5):1375-90.

131. Pattanayek R, Mori T, Xu Y, Pattanayek S, Johnson CH, Egli M. Structures of KaiC circadian clock mutant proteins: a new phosphorylation site at T426 and mechanisms of kinase, ATPase and phosphatase. PLoS One. 2009;4(11):7529.

132. Xu Y, Mori T, Qin X, Yan H, Egli M, Johnson CH. Intramolecular regulation of phosphorylation status of the circadian clock protein KaiC. PLoS One. 2009:4(11):7509.

133. Garces RG, Wu N, Gillon W, Pai EF. Anabaena circadian clock proteins KaiA and KaiB reveal a potential common binding site to their partner KaiC. EMBO J. 2004;23(8):1688-98.

134. Iwase R, Imada K, Hayashi F, Uzumaki T, Morishita M, Onai K, Furukawa Y, Namba K, Ishiura M. Functionally Important Substructures of Circadian Clock Protein KaiB in a Unique Tetramer Complex. J Biol Chem. 2005;280(52):43141-9.

135. Hitomi K, Oyama T, Han S, Arvai AS, Getzoff ED. Tetrameric Architecture of the Circadian Clock Protein KaiB: A novel interface fo intermolecular interactions and its impact on the circadian rhythm. J Biol Chem. 2005;280(19):19127-35

136. Villarreal SA, Pattanayek R, Williams DR, Mori T, Qin X, Johnson CH, Egli M, Stewart PL. CryoEM and Molecular Dynamics of the Circadian KaiB-KaiC Complex Indicates That KaiB Monomers Interact with KaiC and Block ATP Binding Clefts. J Mol Biol. 2013;425(18):3311-24.

137. Pattanayek R, Williams DR, Pattanayek S, Mori T, Johnson CH, Stewart $\mathrm{PL}$, Egli M. Structural model of the circadian clock KaiB-KaiC complex and mechanism for modulation of KaiC phosphorylation. EMBO J. 2008;27(12):1767-78.

138. Loza-Correa M, Sahr T, Rolando M, Daniels C, Petit $P$, Skarina T, Gomez Valero L, Dervins-Ravault D, Honoré N, Savchenko A, Buchrieser C. The Legionella pneumophila kai operon is implicated in stress response and confers fitness in competitive environments. Environ Microbiol. 2014;16(2):359-81.

139. Chang YG, Cohen SE, Phong C, Myers WK, Kim Yl, Tseng R, Lin J, Zhang L, Boyd JS, Lee Y, Kang S, Lee D, Li S, Britt RD, Rust MJ, Golden SS, LiWang A. A protein fold switch joins the circadian oscillator to clock output in cyanobacteria. Science. 2015;349(6245):324-8.

140. Dvornyk V, Knudsen B. Functional divergence of the circadian clock proteins in prokaryotes. Genetica. 2005;124:247-54

141. Neuner A, Jannasch HW, Belkin S, Stetter KO. Thermococcus litoralis sp. nov.: A new species of extremely thermophilic marine archaebacteria. Arch Microbiol. 1990;153(2):205-7.

142. Gonzalez JM, Masuchi Y, Robb FT, Ammerman JW, Maeder DL, Yanagibayashi M, Tamaoka J, Kato C. Pyrococcus horikoshii sp. nov., a hyperthermophilic archaeon isolated from a hydrothermal vent at the Okinawa Trough. Extremophiles. 1998;2:123-30.

143. Elvitigala T, Stöckel J, Ghosh BK, Pakrasi HB. Effect of continuous light on diurnal rhythms in Cyanothece sp. ATCC 51142. BMC Genomics. 2009;10:226.

144. Shi T, Ilikchyan I, Rabouille S, Zehr JP. Genome-wide analysis of diel gene expression in the unicellular $\mathrm{N}_{2}$-fixing cyanobacterium Crocosphaera watsonii WH 8501. ISME J. 2010;4(5):621-32.

145. Golden SS, Ishiura M, Johnson CH, Kondo T. Cyanobacterial Circadian Rhythms. Ann Rev Plant Physiol and Plant Mol Biol. 1997:48:327-54.

146. Chen YB, Zehr JP, Mellon M. Growth and nitrogen fixation of the diazotrophic filamentous nonheterocystous cyanobacterium Trichodesmium sp, IMS 101 in defined media: Evidence for a circadian rhythm. J Phycol. 1996:32(6):916-23.

147. Roenneberg T, Carpenter EJ. Daily rhythm of $\mathrm{O}_{2}$-evolution in the cyanobacterium Trichodesmium thiebautii under natural and constant conditions. Marine Biol. 1993;117(4):693-7.

148. Onai K, Morishita M, Itoh S, Okamoto K, Ishiura M. Circadian Rhythms in the Thermophilic Cyanobacterium Thermosynechococcus elongatus: Compensation of Period Length over a Wide Temperature Range. J Bacteriol. 2004;186(15):4972-977. 
149. Vijayan V, Zuzow R, O'Shea EK. Oscillations in supercoiling drive circadian gene expression in cyanobacteria. Proc Nat Acad Sci USA. 2009;106(52):22564-8.

150. Stöckel J, Welsh EA, Liberton M, Kunnvakkam R, Aurora R, Pakrasi HB. Global transcriptomic analysis of Cyanothece 51142 reveals robust diurnal oscillation of central metabolic processes. Proc Nat Acad Sci USA. 2008;105(16):6156-161.

151. Toepel J, Welsh E, Summerfield TC, Pakrasi HB, Sherman LA. Differential transcriptional analysis of the cyanobacterium Cyanothece sp. strain ATCC 51142 during light-dark and continuous-light growth. J Bacteriol. 2008;190(11):3904-13.

152. Kato H, Watanabe S, Kaori NM, Chibazakura T, Tozawa Y, Yoshikawa H. Exploration of a Possible Partnership among Orphan Two-Component System Proteins in Cyanobacterium Synechococcus elongatus PCC 7942. Biosci, Biotechnol, Biochem. 2012;76(8):1484-91.

\section{Submit your next manuscript to BioMed Central} and we will help you at every step:

- We accept pre-submission inquiries

- Our selector tool helps you to find the most relevant journal

- We provide round the clock customer support

- Convenient online submission

- Thorough peer review

- Inclusion in PubMed and all major indexing services

- Maximum visibility for your research

Submit your manuscript at www.biomedcentral.com/submit 